\title{
A Stiffness-Based Quality Measure for Compliant Grasps and Fixtures
}

\author{
Qiao Lin, Joel W. Burdick, and Elon Rimon
}

\begin{abstract}
This paper presents a systematic approach to quantifying the effectiveness of compliant grasps and fixtures of an object. The approach is physically motivated and applies to the grasping of two- and three-dimensional objects by any number of fingers. The approach is based on a characterization of the frame-invariant features of a grasp or fixture stiffness matrix. In particular, we define a set of frame-invariant characteristic stiffness parameters, and provide physical and geometric interpretation for these parameters. Using a physically meaningful scheme to make the rotational and translational stiffness parameters comparable, we define a frame-invariant quality measure, which we call the stiffness quality measure. An example of a frictional grasp illustrates the effectiveness of the quality measure. We then consider the optimal grasping of frictionless polygonal objects by three and four fingers. Such frictionless grasps are useful in high-load fixturing applications, and their relative simplicity allows an efficient computation of the globally optimal finger arrangement. We compute the optimal finger arrangement in several examples, and use these examples to discuss properties that characterize the stiffness quality measure.
\end{abstract}

Index Terms-Compliance modeling, configuration space, fixturing, grasping, optimization, stiffness.

\section{INTRODUCTION}

C OMPLIANCE can play a dominant role in passive grasps such as workpiece fixturing, and can also be used to model the finger forces in active grasps. This paper presents a frame-invariant quality measure for compliant grasps and fixtures, and considers its application to the planning of compliant grasps and fixtures. To our knowledge, the quality measure presented here provides the first systematic approach to quantifying the effectiveness of compliant (as opposed to rigid) grasps and fixtures. The approach is frame-invariant, applies to the grasping or fixturing of two-dimensional (2-D) and three-dimensional (3-D) objects by any number of fingers, and can be used with any compliance model that yields a grasp stiffness matrix. For the sake of convenience, the term grasping will hereafter also apply to fixturing.

Manuscript received February 17, 1998; revised November 24, 1999. This paper was recommended for publication by Associate Editor P. Allen and Editor A. De Luca upon evaluation of the reviewers' comments. This paper was presented in part at the International Conference on Robotics and Automation, Albuquerque, NM, April 1997.

Q. Lin was with the Division of Engineering and Applied Science, California Institute of Technology, Pasadena, CA 91125 USA. He is now with the Department of Mechanical Engineering, Carnegie Mellon University, Pittsburgh, PA 15213 USA.

J. W. Burdick is with the Division of Engineering and Applied Science, California Institute of Technology, Pasadena, CA 91125 USA.

E. Rimon is with the Department of Mechanical Engineering, Technion, Israel Institute of Technology, Haifa 32000, Israel.

Publisher Item Identifier S 1042-296X(00)10594-4.
Compliant grasps have received considerable attention in the robotic grasping literature. Hanafusa and Asada [1], [] use a linear spring model to find stable three-finger planar grasps. Their work was extended by Nguyen [2], who uses a linear spring model to compute the stiffness matrix of more general grasps. Kumar and his co-workers [3], [4] employ a more sophisticated linear spring compliance model to derive the grasp stiffness matrix and show how the contact geometry influences the grasp stability. In studying compliance in the presence of friction, Cutkosky and Wright [5] note that stability is influenced by initial loading as well as contact geometry. While the linear-spring compliance model has been widely used by roboticists, it is not supported by experiments or results from elasticity theory. Rimon and Burdick [6] use overlap functions to model nonlinear compliance effects. Lin et al. [7] use these overlap functions to compute and analyze the grasp stiffness matrix for various contact models, including the experimentally and theoretically justified Hertz model. The quality measure presented in this paper can be used with a stiffness matrix formula resulting from any compliance model. For the purpose of providing concrete examples, the compliance modeling schemes given in [7], [6], and [3] will be used for frictionless and frictional grasps, respectively.

Prior work on quantifying grasp effectiveness has mostly focused on the rigid-body mechanics of the grasp, while ignoring compliance effects [8]. Let the wrench (i.e., force and torque) due to a unit force applied by a contacting finger be termed a generating wrench. Li and Sastry [9] suggest a quality measure based on the smallest singular value of the grasp matrix, whose columns consist of the generating wrenches. Kirkpatrick et al. [10] define the radius of the maximal ball inscribed in the convex hull of the generating wrenches as a quality measure. This idea is also followed by Ferrari and Canny [11]. However, these quality criteria depend on the choice of coordinate frames - a grasp which is optimal under one choice of reference frame may fail to be optimal under another. Several authors have devised schemes to avoid this problem. Markenscoff and $\mathrm{Pa}-$ padimitriou [12] minimize the worst-case finger forces needed to balance any external unit force acting at a given point of the object. Mirtich and Canny [13] first compute the grasps that best counteract pure forces, and then select among these grasps the one which best resists pure torques. We use a completely different approach to defining the quality measure-while they define a quality measure in a lexicographical manner to avoid comparing forces with torques, we directly address the comparison of rotational and translational stiffnesses (see below). Teichmann [14] suggests as a quality measure the largest inscribed ball (as defined in [10]) with respect to all choices of coordinate 
frames. However, his approach applies only to rigid grasps, and appears to lack adequate physical interpretation. In fact, all of these prior works address only rigid grasps, while we explicitly include compliance in our analysis.

Also focusing on grasps consisting of perfectly rigid bodies, several authors have evaluated grasp quality using the margin by which grasp contact constraints are satisfied. Ji and Roth [15] minimized the dependence on friction of the equilibrium finger forces, while Trinkle [16] investigated the smallest of the normalized equilibrium finger forces. Other quality measures along this line include those proposed by Kerr and Roth [17], Chen et al. [18], Bicchi [19], and Varma and Tasch [20]. In related work, Nakamura et al. [21] considered the largest allowable dynamic perturbations to the contact point position that cause no slippage of the fingers relative to the object.

The potentially important role played by compliance in many grasping and fixturing operations calls for the development of quality measures that take these effects into consideration. However, quality measures for compliant grasps and fixtures are rather scarce. While the quality measure proposed by Ponce et al. [22] applies to three-finger immobilizing rigid grasps, it offers insight into the rotational stiffness of such grasps when compliance is introduced. Prattichizzo et al. [23] defined robustness measures that quantify a compliant grasp's sensitivity to perturbations of a given work load. Cutkosky and Kao [24] pointed out that the eigenvalues of the grasp stiffness matrix provides information on grasp stability. Donoghue et al. [3] proposed using a weighted square sum of the displacement components of a fixtured object induced by a given applied wrench. However, both stiffness matrix eigenvalues and the square sum of the displacement components are dependent upon the choice of reference frames.

This paper builds on prior work and presents a frame-invariant quality measure that is practically useful due to its interesting physical meanings. We first identify the frame-invariant features of a grasp's stiffness matrix, in terms of invariant scalars called the principal translational and rotational stiffness parameters (Section III). These parameters were first identified by Patterson and Lipkin [25] using screw theory. The same parameters were obtained by us using a different approach, and were proven by us to have frame invariant properties. We discuss the relation between the two approaches, and provide a novel geometrical interpretation of the principal stiffness parameters. Once the invariant stiffness parameters are identified, we turn to the issue of how to incorporate these parameters in a physically meaningful way into a single quality measure (Section IV). The key to our approach is the conversion of the rotational stiffness parameters into equivalent translational stiffnesses according to considerations of the object's deflection and equivalence of elastic energy. Based on this method, we define a stiffness quality measure in terms of the principal stiffness parameters (Section IV). Examples are used to illustrate the applicability of the approach. Furthermore, we compare our results against the grasp quality measure of Mirtich and Canny [13] to illustrate why consideration of compliance effects can be important for some applications.

Our quality measure can be used as the basis for optimal grasp or fixture planning by optimizing the quality measure on the space of feasible grasps. We consider in detail the optimal grasping of frictionless polygonal objects by three and four fingers. Such grasps are practically important for fixturing applications involving large forces, where contact friction should not be relied upon for object immobilization [26]. For this limited class of grasps, it is possible to compute the globally optimal finger arrangement with an efficient method described in this paper. Furthermore, in some cases, it is possible to find the optimal grasp by inspection. We apply these tools to several examples and draw conclusions as to which properties characterize the optimal finger arrangements.

\section{BACKGROUND AND REVIEW OF COMPLIANCE MODELING}

This section introduces relevant terminology and reviews the modeling of compliance in grasps and fixtures. To use our quality measure, one must be able to derive a stiffness matrix for a given grasp or fixture. The sophistication of the compliance model used to derive the stiffness matrix will depend upon the requirements of a given application, and the model may include such effects as friction (e.g., Coulomb friction) at the contacts, nonlinear elasticity, and the curvatures of the contacting surfaces. Since our quality measure does not depend on a particular compliance model, it suffices to review some basic facts regarding compliance modeling.

A grasp or fixture arrangement consists of an object $\mathcal{B}$ contacted by $n$ fingers $\mathcal{A}_{1}, \ldots, \mathcal{A}_{n}$. We first assume that the bodies are quasi-rigid. That is, deformations due to compliance effects are localized to the vicinity of the contacts, so that the overall motion of $\mathcal{B}$ relative to $\mathcal{A}_{i}$ can be described using rigid-body kinematics. Any compliance modeling approach that uses a lumped parameter model of compliance at the contacts, such as the widely used linear spring model [4], implicitly relies upon the quasi-rigid assumption. Elasticity theory [27] suggests that this is an excellent assumption, provided that the bodies do not involve slender substructures. Second, since we are interested in the motion of $\mathcal{B}$ relative to $\mathcal{A}_{i}$, the fingers are assumed to be stationary.

Since the fingers are stationary, we can focus on $\mathcal{B}$ 's configuration space, which is defined as follows. Let $\mathcal{F}_{W}$ be a stationary world reference frame, and $\mathcal{F}_{B}$ a frame fixed to $\mathcal{B}$. A configuration of $\mathcal{B}$ is a pair $q=(d, R)$, where $d \in \mathbb{R}^{3}$ is the position, and $R \in S O(3)$ the orientation of $\mathcal{F}_{B}$ relative to $\mathcal{F}_{W}$. The set of all configurations, denoted $\mathcal{C}$, is $\mathcal{B}$ 's configuration space (c-space). To parametrize $\mathcal{C}$ in hybrid coordinates, we parametrize $S O(3)$ by $R(\theta)=\exp (\hat{\theta})$, where $\hat{\theta}$ is the skew-symmetric matrix such that $\hat{\theta} x=\theta \times x$ for any $x \in \mathbb{R}^{3}$. The tangent space to $\mathcal{C}$ at $q$, denoted $T_{q} \mathcal{C}$, is the set of all tangent vectors, or velocities of $\mathcal{B}$, at $q$. Tangent vectors represent instantaneous displacements of $\mathcal{B}$ and can approximate small displacements. ${ }^{1}$ In hybrid coordinates, tangent vectors take the form $\dot{q}=(v, \omega)$, where $v$ is the linear velocity of $\mathcal{F}_{B}$ 's origin, and $\omega$ is the angular velocity of $\mathcal{F}_{B}$. The wrench space at $q$, denoted $T_{q}^{*} \mathcal{C}$, is the set of all wrenches (or covectors) acting on $\mathcal{B}$ at $q$. A wrench takes the

\footnotetext{
${ }^{1}$ While customarily called rigid body velocities, tangent vectors can represent both physical velocities $\dot{q}$ (rigid displacement per unit time) and infinitesimal rigid displacements $\dot{q} d t$ ( $d t$ is an infinitesimal time interval). In this paper, we use the latter interpretation and drop $d t$ for notational convenience.
} 
form $\boldsymbol{w}=(f, \tau)$ in hybrid coordinates, where $f$ is the force acting on $\mathcal{B}$ and $\tau$ is the torque. When $\mathcal{B}$ is planar, c-space can be parameterized with $q=(d, \theta) \in \mathbb{R}^{2} \times \mathbb{R}$, by choosing the $z$-axes of $\mathcal{F}_{W}$ and $\mathcal{F}_{B}$ orthogonal to the plane.

Consider an alternative choice of world frame $\overline{\mathcal{F}}_{W}$, displaced from $\mathcal{F}_{W}$ by $\left(d_{w}, R_{w}\right)$, and another object frame $\overline{\mathcal{F}}_{B}$, displaced from $\mathcal{F}_{B}$ by $\left(d_{b}, R_{b}\right)$. A configuration with coordinates $q$ will have new coordinates $\bar{q}$ with respect to $\overline{\mathcal{F}}_{W}$ and $\overline{\mathcal{F}}_{B}$. Let $T$ denote the transformation between the two coordinates, $q=$ $T(\bar{q})$. Then $T$ is a linear mapping, and the transformation matrix for the 3-D and 2-D cases is given by

$$
T_{6 \times 6}=\left(\begin{array}{cc}
R_{w} & \hat{R_{0}} d_{b} R_{w} \\
0 & R_{w}
\end{array}\right) \quad T_{3 \times 3}=\left(\begin{array}{cc}
R_{w} & J R_{0} d_{b} \\
0 & 1
\end{array}\right)
$$

where $J=\left(\begin{array}{rr}0 & 1 \\ -1 & 0\end{array}\right)$ and $R_{0}$ is the orientation of $\mathcal{F}_{B}$ relative to $\mathcal{F}_{W}$ at $q_{0}$. Since $d_{w}$ and $R_{b}$ do not appear in $T$, a translation of $\mathcal{F}_{W}$ or a rotation of $\mathcal{F}_{B}$ do not affect the transformation $T$. The tangent and cotangent vectors transform as follows [28]:

$$
\dot{\bar{q}}=T^{-1} \dot{q} \quad \overline{\boldsymbol{w}}=T^{T} \boldsymbol{w}
$$

where $\dot{q}$ and $\boldsymbol{w}$ are tangent and cotangent vectors in the $q$-coordinates, and $\dot{\bar{q}}$ and $\overline{\boldsymbol{w}}$ are tangent and cotangent vectors in the $\bar{q}$-coordinates.

To derive the grasp stiffness matrix, one defines an elastic potential energy function $\Pi(q)$ that describes the amount of energy stored in the finger/object system due to deformation of the contacting bodies. Once $\Pi(q)$ is known, the gradient vector $-\nabla \Pi(q)$ gives the net wrench acting on $\mathcal{B}$ due to the finger forces. The fingers form an equilibrium grasp if (in the absence of any external wrench) the finger contact forces produce a zero net wrench on $\mathcal{B}$. Thus, an equilibrium grasp at $q_{0}$ is characterized by the condition $\nabla \Pi\left(q_{0}\right)=0$. The grasp's stiffness matrix is the Hessian, $K=D^{2} \Pi\left(q_{0}\right)$. Since $\nabla \Pi\left(q_{0}\right)=0$ at an equilibrium grasp, the behavior of $\Pi$ in the vicinity of $q_{0}$ is determined by $K$. When subjected to an arbitrary external disturbance, $\mathcal{B}$ may be displaced from $q_{0}$. The grasp is said to be (asymptotically) stable if $\mathcal{B}$ returns to $q_{0}$ after the external disturbance is removed. If $K$ is positive definite then $q_{0}$ is a local minimum point of $\Pi$, and the grasp is stable [6]. We consider generic stable grasps, whose stiffness matrix is positive definite, and refer to these grasps simply as stable grasps.

The stiffness matrix of a stable grasp has the following wellknown interpretation. When an object $\mathcal{B}$, held in an equilibrium grasp, is subjected to a disturbing wrench $\boldsymbol{w}_{\text {ext }}$, it undergoes a displacement. Provided that the displacement is sufficiently small, it can be approximated by a tangent vector, or infinitesimal displacement, $\dot{q} \in T_{q_{0}} \mathcal{C}$. The stiffness matrix relates the displacement of $\mathcal{B}$ to the net wrench generated by the fingers' reaction forces, according to the formula $\boldsymbol{w}=-K \dot{q}$. When $\mathcal{B}$ is subjected to an external wrench $w_{\text {ext }}$, the finger/object system settles at a nearby equilibrium where the fingers' reaction wrench balances the external wrench. Thus, the stiffness matrix relates the displacement of $\mathcal{B}$ to the external wrench by the formula $w_{\text {ext }}=K \dot{q}$. Its positive definite inverse, $C=K^{-1}$, is called the grasp compliance matrix, and it establishes the rela- tionship $\dot{q}=C \boldsymbol{w}_{\text {ext }}$. Using formula (2), it can be readily shown that the stiffness matrix obeys the transformation law

$$
\bar{K}=T^{T} K T
$$

where $\bar{K}$ is the stiffness matrix associated with new world and body frames $\overline{\mathcal{F}}_{W}$ and $\overline{\mathcal{F}}_{B}$.

\section{PRINCIPAL STIFFNESS PARAMETERS}

In this section, we identify certain frame-invariant parameters of compliant grasps. These parameters are based on the grasp stiffness matrix $K$ and the compliance matrix $C=K^{-1}$. Recall that a wrench acting on $\mathcal{B}$ and the corresponding infinitesimal displacement of $\mathcal{B}$ are related by $\boldsymbol{w}=K \dot{q}$, or $\dot{q}=C \boldsymbol{w}$. We use the following partition of $K$ and $C$ :

$$
K=\left(\begin{array}{ll}
K_{11} & K_{12} \\
K_{12}^{T} & K_{22}
\end{array}\right) \quad C=\left(\begin{array}{ll}
C_{11} & C_{12} \\
C_{12}^{T} & C_{22}
\end{array}\right)
$$

where the entries $K_{i j}$ are $3 \times 3$ matrices in the 3 -D case. In the 2-D case, the dimensions of $K_{11}, K_{12}$, and $K_{22}$ become $2 \times 2,2 \times 1$, and $1 \times 1$, respectively. Given any tangent vector $\dot{q}=(v, \omega)$, we use the notation $(K \dot{q})_{1}=K_{11} v+K_{12} \omega$ and $(K \dot{q})_{2}=K_{12}^{T} v+K_{22} \omega$. Similarly, we write $(C \boldsymbol{w})_{1}=C_{11} f+$ $C_{12} \tau$ and $(C w)_{2}=C_{12}^{T} f+C_{22} \tau$ for any wrench $\boldsymbol{w}=(f, \tau)$.

\section{A. Definition of the Principal Stiffness Parameters}

The eigenvalues of $K$ could provide important insight into the stiffness matrix structure. However, while the sign of these eigenvalues is preserved under changes of coordinate frames, their magnitude is not frame-invariant. To circumvent this difficulty, we describe a subspace of tangent vectors on which $K$ has a frame-invariant structure. This subspace is given by

$$
V=\left\{\dot{q} \in T_{q_{0}} \mathcal{C}: f=(K \dot{q})_{1}=0\right\}
$$

where $q_{0}$ is the equilibrium grasp configuration of $\mathcal{B}$. In words, the subspace $V$ consists of small displacements of $\mathcal{B}$ which cause the fingers to react with a pure net torque on $\mathcal{B}$. Using the partition of $K$, we obtain $V=\left\{(v, \omega): v=-K_{11}^{-1} K_{12} \omega\right\}$, from which it follows that $V$ can be parametrized in terms of $\omega \in \mathbb{R}^{3}$ as $^{2}$

$$
V=\left\{\dot{q}=P \omega: \omega \in \mathbb{R}^{3}\right\}, \quad \text { where } P=\left(\begin{array}{c}
-K_{11}^{-1} K_{12} \\
I
\end{array}\right) \text {. }
$$

Let $K_{V}$ denote the restriction of the stiffness matrix $K$ to the subspace $V$. We now derive an expression for $K_{V}$. The stiffness matrix $K$ is a linear operator from $T_{q_{0}} \mathcal{C}$ to $T_{q_{0}}^{*} \mathcal{C}$, and $\dot{q}^{T} K \dot{q}$ is consequently a symmetric bilinear operator on $T_{q_{0}} \mathcal{C}$. Since the vectors in $V$ are parametrized by $\omega$, we have from (5) that $\omega^{T} K_{V} \omega=\omega^{T} P^{T} K P \omega$ for arbitrary $\omega$. Thus $K_{V}$ has the representation $K_{V}=P^{T} K P=K_{22}-K_{12}^{T} K_{11}^{-1} K_{12}$. In addition, the pure torque corresponding to $\dot{q} \in V$ is given by $(K \dot{q})_{2}=K_{V} \omega$.

Consider now two new frames $\overline{\mathcal{F}}_{W}$ and $\overline{\mathcal{F}}_{B}$, with overbars denoting terms associated with these frames. The linear operator $K_{V}$ has the following invariance property.

\footnotetext{
${ }^{2} K_{11}$ is invertible since, in general, the principal submatrices of a positive definite matrix are positive definite and therefore invertible.
} 
Proposition 1: Let $V$ and $\bar{V}$ be the subspaces parametrized by (5) in the $q$ and $\bar{q}$ coordinates. Let $K_{V}$ and $\bar{K}_{V}$ be the restriction of the respective stiffness matrix to $V$ and $\bar{V}$. Then $K_{V}$ and $\bar{K}_{V}$ obey the orthogonal transformation $\bar{K}_{V}=R_{w}^{T} K_{V} R_{w}$, where $R_{w}$ is the rotation matrix from $\mathcal{F}_{W}$ to $\overline{\mathcal{F}}_{W}$. Hence, the eigenvalues of $K_{V}$ are frame-invariant.

Proof: Using the stiffness matrix transformation rule (3) and formula (1) for $T$, the components of the stiffness matrix $\bar{K}$ are: $\bar{K}_{11}=R_{w}^{T} K_{11} R_{w}, \bar{K}_{12}=R_{w}^{T}\left(K_{12}+K_{11} \hat{R_{0}} d_{b}\right) R_{w}$, and $\bar{K}_{22}=R_{w}^{T}\left(K_{22}+K_{12}^{T} \hat{R_{0}} d_{b}-\hat{R_{0} d_{b} K_{12}}-\hat{R_{0}} d_{b} K_{11} \hat{R_{0}} d_{b}\right) R_{w}$. Substituting these expressions into $\bar{K}_{V}=\bar{K}_{22}-\bar{K}_{12}^{T} \bar{K}_{11}^{-1} \bar{K}_{12}$ gives $\bar{K}_{V}=R_{w}^{T} K_{V} R_{w}$.

Dually, there also exists a subspace of wrenches on which the compliance matrix $C$ has a frame-invariant structure. This subspace is given by

$$
W=\left\{\boldsymbol{w} \in T_{q_{0}}^{*} \mathcal{C}: \omega=(C \boldsymbol{w})_{2}=0\right\} .
$$

The subspace $W$ thus consists of external wrenches that cause the grasped object $\mathcal{B}$ to move with pure translation. The subspace $W$ can be parametrized in terms of $f \in \mathbb{R}^{3}$ as

$$
W=\left\{\boldsymbol{w}=Q f: f \in \mathbb{R}^{3}\right\}, \quad \text { where } Q=\left(\begin{array}{c}
I \\
-C_{22}^{-1} C_{12}^{T}
\end{array}\right) .
$$

Using this parametrization, the restriction of $C$ to the subspace $W$, denoted $C_{W}$, takes the form $C_{W}=Q^{T} C Q=C_{11}-$ $C_{12} C_{22}^{-1} C_{12}^{T}$. Moreover, for any wrench $\boldsymbol{w} \in W$, the resulting pure-translation is given by $v=(C w)_{1}=C_{W} f$.

By again considering two new frames $\overline{\mathcal{F}}_{W}$ and $\overline{\mathcal{F}}_{B}$, we can show the following invariance property of $C_{W}$ in a way similar to the proof of Proposition 1.

Proposition 2: Let $W$ and $\bar{W}$ be the subspaces parametrized by (6) in the $q$ and $\bar{q}$ coordinates. Let $C_{W}$ and $\bar{C}_{W}$ be the restriction of the respective compliance matrix to $W$ and $\bar{W}$. Then $C_{W}$ and $\bar{C}_{W}$ obey the orthogonal transformation $\bar{C}_{W}=$ $R_{w}^{T} C_{W} R_{w}$, where $R_{w}$ is the rotation matrix from $\mathcal{F}_{W}$ to $\overline{\mathcal{F}}_{W}$. Hence, the eigenvalues of $C_{W}$ are frame-invariant.

Propositions 1 and 2 lead to the following observations. The behavior of $K$ on the tangent subspace $V$ characterizes the rotational stiffness of the grasp. In response to an instantaneous displacement in $V$, the reaction wrench is a pure torque. In addition, the reaction torque varies by at most a pure rotation corresponding to different choices of frames. Similarly, the behavior of $C$ on the wrench subspace $W$ characterizes the translational compliance of the grasp. A wrench in $W$ generates a pure translation of $\mathcal{B}$, which is the same up to rotation with respect to different frames. We also note that by the inversion formula of a partitioned matrix, ${ }^{3} C_{W}=K_{11}^{-1}$. Based on these observations, we define the frame-invariant principal parameters of $K$ as follows.

Definition 1: Let $K$ be the grasp stiffness matrix, and $C=$ $K^{-1}$ the grasp compliance matrix. Let $K_{V}$ and $C_{W}$ be the re-

$$
\text { } \begin{aligned}
{ }^{3} \text { That is }\left(\begin{array}{ll}
K_{11} & K_{12} \\
K_{12}^{T} & K_{22}
\end{array}\right) & =\left(\begin{array}{ll}
C_{11} & C_{12} \\
C_{12}^{T} & C_{22}
\end{array}\right)^{-1} \\
& =\left(\begin{array}{ccc} 
& C_{W}^{-1} & -C_{11}^{-1} C_{12} \Delta^{-1} \\
-\Delta^{-1} C_{12}^{T} C_{11}^{-1} & \Delta^{-1}
\end{array}\right)
\end{aligned}
$$

where $\Delta=C_{22}-C_{12}^{T} C_{11}^{-1} C_{12}$. striction of $K$ and $C$ to the subspaces $V$ and $W$. Then the principal rotational stiffnesses of the grasp are the eigenvalues $\mu_{i}$ $(i=1,2,3)$ of $K_{V}$ (where $K_{V}=K_{22}-K_{12}^{T} K_{11}^{-1} K_{12}$ ), and the principal translational stiffnesses of the grasp are the eigenvalues $\sigma_{i}(i=1,2,3)$ of $C_{W}^{-1}=K_{11}$.

For planar grasps, the principal stiffness parameters have the following well-known physical interpretation. It can be shown that every planar grasp has a unique location of an object frame origin, given by

$$
d_{b}=R_{0}^{T} J K_{11}^{-1} K_{12}
$$

such that when $\overline{\mathcal{F}}_{B}$ is placed at this location, $\bar{K}_{3 \times 3}$ takes the block-diagonal form $\bar{K}=\operatorname{diag}\left(R_{w}^{T} K_{11} R_{w}, \mu\right)$. That is, for planar grasps the translational and rotational effects are decoupled about this special point, called the center of compliance [2]. The principal translational and rotational stiffnesses of a planar grasp are physically the translational and rotational stiffnesses about the center of compliance. For 3-D grasps, there is generally no such center of compliance, and the stiffness matrix in general cannot be made block-diagonal. However, it is important to note that the principal stiffness parameters are still well defined in the 3-D case.

\section{B. Screw Coordinates Interpretation}

While searching for a 3-D analog of the center of compliance, Patterson and Lipkin [25] were the first to recognize the existence of the principal stiffness parameters. In order to provide a sense of continuity with the existing literature, we show that our principal parameters are equivalent to the ones derived by Patterson and Lipkin using screw coordinates. It is worth noting that we have derived these parameters independently using hybrid coordinates. Moreover, to our knowledge we have provided the first formal analysis of the frame-invariance properties of the principal stiffness parameters.

The screw coordinates of a tangent vector $\dot{q}=(v, \omega)$ consist of a screw axis and two scalars called pitch and magnitude. If $\omega \neq 0$, the instantaneous screw axis of $\dot{q}$ is the line parallel to $\omega$ which passes through the point $\omega \times v /\|\omega\|^{2}$. The pitch of $\dot{q}$ is $(\omega \cdot v) /\|\omega\|^{2}$, and its magnitude is $\|\omega\|$. If $\omega=0$, the pitch is infinite, the magnitude is $\|v\|$, and the screw axis is the line parallel to $v$ which passes through the origin. Similarly, the screw coordinates of a wrench $w=(f, \tau)$ consist of an axis, pitch, and magnitude. If $f \neq 0$, the screw axis of the wrench is the line parallel to $f$ which passes through the point $f \times \tau /\|f\|^{2}$. The pitch of $w$ is $(f \cdot \tau) /\|f\|^{2}$, and its magnitude is $\|f\|$. When $f=0$, the wrench has infinite pitch, its magnitude is $\|\tau\|$, and its axis is the line parallel to $\tau$ which passes through the origin.

Consider an infinitesimal displacement $\dot{q}_{i}=P \omega_{i} \in V$, where $\omega_{i}$ is a unit eigenvector of $K_{V}$ associated with the eigenvalue $\mu_{i}$. Correspondingly, $\mathcal{B}$ experiences a pure torque given by $\tau=\left(K \dot{q}_{i}\right)_{2}=\mu_{i} \omega_{i}$. It follows that the displacement $\dot{q}_{i}$ induces a pure-torque wrench of magnitude $\mu_{i}$ about the screw axis of $\dot{q}_{i}$. On the other hand, for $\boldsymbol{w}_{i}=Q f_{i} \in W$ where $f_{i}$ is a unit eigenvector of $C_{W}$ associated with the eigenvalue $\sigma_{i}^{-1}$, we have $v=\left(C \boldsymbol{w}_{i}\right)_{1}=\sigma_{i}^{-1} f_{i}$. Hence, the wrench $\boldsymbol{w}_{i}$ generates a pure-translation displacement of magnitude $\sigma_{i}^{-1}$ along the screw axis of $w_{i}$. We can now interpret the principal stiffness 
parameters in terms of screw coordinates. One can associate with every stiffness matrix $K$ six frame-invariant screw axes. A displacement of $\mathcal{B}$ along the first three axes results in a pure torque which acts on $\mathcal{B}$ along the same axis, of magnitude which is determined by the rotational stiffness $\mu_{i}$ for $i=1,2,3$. A wrench applied to $\mathcal{B}$ along the other three axes results in a pure translation of $\mathcal{B}$ along the same axis, and the magnitude of the translation is determined by the reciprocals of the translational stiffness $1 / \sigma_{i}$ for $i=1,2,3$.

\section{Geometric Interpretation}

We now interpret the principal stiffness parameters in terms of the geometry of two level-sets that are related to the elastic energy that is induced by compliant deformations. The first is a level-set in the tangent space, defined by $\mathcal{S}=\left\{\dot{q} \in T_{q_{0}} \mathcal{C}: \Phi(\dot{q})=1\right\}$, where $\Phi(\dot{q})=(1 / 2) \dot{q}^{T} K \dot{q}$. The second is a level-set in the wrench space, defined by $\mathcal{T}=\left\{\boldsymbol{w} \in T_{q_{0}}^{*} \mathcal{C}: \Psi(\boldsymbol{w})=1\right\}$, where $\Psi(\boldsymbol{w})=(1 / 2) \boldsymbol{w}^{T} C \boldsymbol{w}$. These level sets consist of tangent vectors or wrenches that induce unit elastic energy due to the associated deformation. Geometrically, these surfaces represent a five-dimensional ellipsoidal surface in the six-dimensional tangent or wrench space. While the shape of these surfaces varies as different coordinate frames are used, they possess frame-invariant features which correspond to the principal stiffness parameters.

First consider the level-set $\mathcal{S}$. For each fixed $\omega$, the subset of $\mathcal{S}$ with this particular value of $\omega$ is denoted $\mathcal{S}_{\omega}$. Each subset $\mathcal{S}_{\omega}$ is a level-set of the function $\Phi_{\omega}(v) \triangleq \Phi(v, \omega)$, in which $\omega$ is a fixed parameter. Rewriting $\Phi(v, \omega)$ as a function of $v$ only gives

$$
\begin{aligned}
\Phi_{\omega}(v)= & \frac{1}{2}\left(v+K_{11}^{-1} K_{12} \omega\right)^{T} K_{11}\left(v+K_{11}^{-1} K_{12} \omega\right) \\
& +\frac{1}{2} \omega^{T} K_{V} \omega .
\end{aligned}
$$

Hence, for each fixed $\omega$, the level-set $\mathcal{S}_{\omega}=\left\{v: \Phi_{\omega}(v)=1\right\}$ is a 2-D ellipsoidal surface with principal semi-axes of lengths $\left(\left(2-\omega^{T} K_{V} \omega\right) / \sigma_{i}\right)^{1 / 2}(i=1,2,3)$. Since the quadratic form $\omega^{T} K_{V} \omega$ is frame-invariant, these lengths are frame-invariant. When $\omega=0$, these lengths are $\sqrt{2 / \sigma_{i}}(i=1,2,3)$. Thus, the principal translational stiffnesses $\sigma_{i}$ determine the frameinvariant shape of the intersection of $\mathcal{S}$ with the subspace of $T_{q} \mathcal{C}$ defined by $\omega=0$. This geometric interpretation is depicted in Fig. 1.

The level-set $\mathcal{S}$ possesses another frame-invariant geometrical feature. Consider the projection, denoted $\Pi \mathcal{S}$, of the set $\mathcal{S}$ onto the subspace given by $v=0$. Then the boundary of $\Pi \mathcal{S}$ (the silhouette of $\mathcal{S}$ along the direction of projection) is the projection of those points on $\mathcal{S}$ whose surface normal to $\mathcal{S}$ has zero $v$-components. This latter set is denoted $\mathcal{S}_{n}$, and is determined by the condition $(\nabla \Phi(\dot{q}))_{1}=0$. This condition implies that

$$
\begin{aligned}
\mathcal{S}_{n} & =\left\{(v, \omega) \in \mathcal{S}: v=-K_{11}^{-1} K_{12} \omega\right\} \\
& =\left\{(v, \omega) \in \mathcal{S}: \frac{1}{2} \omega^{T} K_{V} \omega=1\right\} .
\end{aligned}
$$

Thus, the projection of $\mathcal{S}$ onto the subspace $v=0$ is given by

$$
\Pi \mathcal{S}=\left\{(v, \omega): v=0 \quad \text { and } \quad \frac{1}{2} \omega^{T} K_{V} \omega \leqslant 1\right\} .
$$

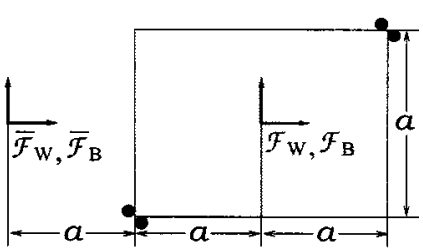

(a)

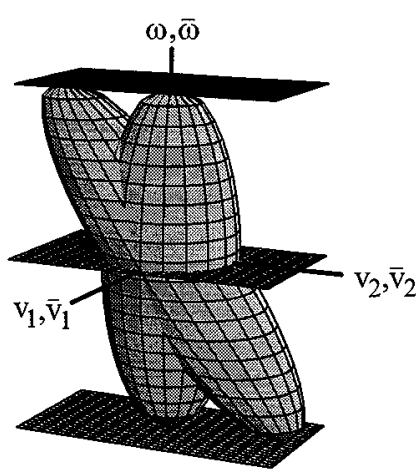

(b)

Fig. 1. (a) Four-finger grasp of a rectangle. (b) The elastic energy ellipsoid in $T_{q_{0}} \mathcal{C}$

The projection set is a 3-D ellipsoid with principal semi-axes of length $\sqrt{2 / \mu_{i}}$, where $\mu_{i}(i=1,2,3)$ are the frame-invariant eigenvalues of $K_{V}$. That is, the principal rotational stiffness parameters are the semi-axis lengths of the ellipsoid formed by projecting $\mathcal{S}$ onto the pure-rotation subspace given by $v=0$.

These ideas are readily visualized for planar grasps, where the level set $\mathcal{S}$ is a 2 -D ellipsoidal surface. Fig. 1(b) shows two such ellipsoids for the four-finger frictionless grasp of a rectangle shown in Fig. 1(a). Fig. 1(b) is a superposition of the coordinates of $\dot{q}$ and $\dot{\bar{q}}$ corresponding to the two choices of frames shown in Fig. 1(a). The upright ellipsoid corresponds to the frames $\mathcal{F}_{W}$ and $\mathcal{F}_{B}$, while the slanted ellipsoid corresponds to the frames $\overline{\mathcal{F}}_{W}$ and $\overline{\mathcal{F}}_{B}$. The frames $\mathcal{F}_{W}$ and $\mathcal{F}_{B}$, as well as $\overline{\mathcal{F}}_{W}$ and $\overline{\mathcal{F}}_{B}$, are coincident. As the figure illustrates, the principal semi-axes of each horizontal cross section of $\mathcal{S}$ have frame invariant lengths. Similarly, the projection of $\mathcal{S}$ onto the $\omega$-axis is bounded by two points, whose $\omega$-coordinates are $\pm \sqrt{2 / \mu}$. These two points are frame invariant, and $\mathcal{S}$ is always bounded by the two horizontal planes $\omega= \pm \sqrt{2 / \mu}$.

The analogous frame-invariant features of the level set $\mathcal{T}$ can be summarized as follows. Each subset of $\mathcal{T}$ with a fixed value of $f$, denoted $\mathcal{T}_{f}$, is a 2 -D ellipsoidal surface whose principal semi-axes have the frame-invariant lengths equal to $\left(\mu_{i}\left(2-f^{T} K_{11}^{-1} f\right)\right)^{1 / 2}(i=1,2,3)$. When $f=0$, the principal semi-axes of $\mathcal{T}_{f}$ have lengths $\sqrt{2 \mu_{i}}$. The projection of $\mathcal{T}$ onto the wrench subspace defined by $\tau=0$ is given by

$$
\Pi \mathcal{T}=\left\{(f, \tau): \tau=0 \quad \text { and } \quad \frac{1}{2} f^{T} K_{11}^{-1} f \leqslant 1\right\}
$$

which is a 3-D ellipsoid whose principal semi-axes have frameinvariant lengths of $\sqrt{2 \sigma_{i}}(i=1,2,3)$.

For the same grasp in Fig. 1(a), the frame-invariant features of $\mathcal{T}$ are shown in Fig. 2, where the upright and slanted ellipsoids again correspond to the frames $\mathcal{F}_{W}$ and $\mathcal{F}_{B}$, and $\overline{\mathcal{F}}_{W}$ and $\overline{\mathcal{F}}_{B}$, respectively. Note that the ellipsoid $\mathcal{T}$ intersects the $\tau$-axis 


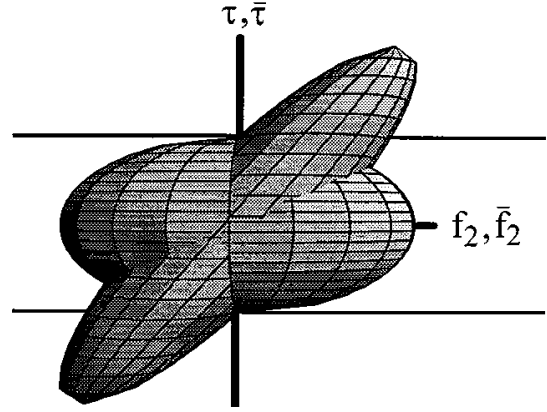

(a)

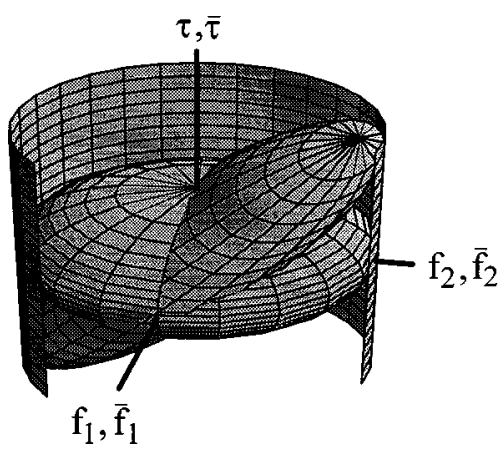

(b)

Fig. 2. The elastic energy ellipsoid $\mathcal{T}$ in $T_{q_{0}}^{*} \mathcal{C}$. (a) $\mathcal{T}$ intersects the $\tau$-axis at the same points $\tau= \pm(2 \mu)^{1 / 2}$. (b) $\mathcal{T}$ is inscribed in the same elliptic cylinder.

at two points whose $\tau$-coordinates are $\pm \sqrt{2 \mu}$ [Fig. 2(a)]. Regardless of frame choices, the horizontal projection of $\mathcal{T}$ is the planar ellipse $(1 / 2) f^{T} K_{11}^{-1} f \leqslant 1$. Hence, with respect to arbitrarily chosen coordinate frames, $\mathcal{T}$ is always inscribed in the vertical cylinder whose base set is this ellipse [Fig. 2(b)].

We also note that the volume of the ellipsoids is frame-invariant, since the volume is determined by $\operatorname{det}(K)$ which is frame-invariant [9]. However, we make no use of the volume in our stiffness quality measure.

\section{A Frame-InVARIANT QUALITY MEASURE}

This section defines a frame-invariant quality measure for compliant grasps based on the principal stiffness parameters. First we must find a way to meaningfully compare the translational and rotational stiffnesses of a grasp. Our approach is based on the notion of object deflection and the elastic energy associated with this deflection.

Let $\dot{q}=\alpha(v, \omega)$ be an infinitesimal displacement of $\mathcal{B}$, where $\alpha$ is a scalar, $\|\omega\|=1$ if $\omega \neq 0$, and $\|v\|=1$ if $\omega=0$. We define the deflection of $\mathcal{B}$ due to the displacement $\dot{q}$ as the maximal displacement of any point in $\mathcal{B}$. Since $\mathcal{B}$ has bounded dimension, such a displacement always exists and is independent of frame choice. If $\omega=0$, the deflection of $\mathcal{B}$ is $|\alpha|$. If $\omega \neq 0$, let $\rho_{\max }(\dot{q})$ be the greatest distance from the instantaneous screw axis associated with $\dot{q}$ to $\mathcal{B}$ 's boundary points. Then $\mathcal{B}$ 's deflection is $|\alpha|\left(\rho_{\max }(\dot{q})^{2}+(v \cdot \omega)^{2}\right)^{1 / 2}$, where $v \cdot \omega$ is the pitch of $\dot{q}$. For planar grasps the instantaneous screw axis is perpendicular to the plane, at a point called the instantaneous center of rotation. In this case $\omega$ is perpendicular to $v$, and the deflection of $\mathcal{B}$ is $|\alpha| \rho_{\max }(\dot{q})$, where $\rho_{\max }(\dot{q})$ is the maximal distance from $\mathcal{B}$ 's instantaneous center of rotation to its boundary points.

We now convert the rotational stiffnesses to equivalent translational stiffnesses using the notion of object deflection. First consider planar grasps, where there is only a single principal rotational stiffness parameter, denoted $\mu$. To compare $\mu$ with the translational stiffness parameters $\sigma_{1}$ and $\sigma_{2}$, we define a parameter which has the units of translational stiffness and whose equivalence with the principal rotational stiffness $\mu$ is determined as follows. As discussed in Section III, $\mu$ is associated with rotations of $\mathcal{B}$ about the grasp's center of compliance. Corresponding to a rotation of $\mathcal{B}$ with magnitude $\alpha$ about the center of compliance, $\mathcal{B}$ 's deflection is $|\alpha| \rho_{\max }$ where $\rho_{\max }$ is the maximum distance from the center of compliance to the boundary of $\mathcal{B}$. The amount of elastic energy induced by this deflection is $(1 / 2) \mu \alpha^{2}$. Suppose that $\mathcal{B}$ undergoes a pure translation of distance $|\alpha| \rho_{\max }$, which results in the same deflection. During this translation, imagine that $\mathcal{B}$, instead of being grasped by the fingers, is attached to a linear spring aligned with the direction of translation. We define the constant of this spring, denoted $\mu_{\mathrm{eq}}$, as equivalent to the principal rotational stiffness $\mu$, if the elastic energy of the linear spring resulting from the (imaginary) translation equals $(1 / 2) \mu \alpha^{2}$. Thus, from the equation $(1 / 2) \mu_{\mathrm{eq}}\left(\rho_{\max } \alpha\right)^{2}=(1 / 2) \mu \alpha^{2}$, we obtain the following expression for the equivalent stiffness $\mu_{\mathrm{eq}}$ :

$$
\mu_{\mathrm{eq}}=\frac{\mu}{\rho_{\max }^{2}} .
$$

The parameter $\mu_{\mathrm{eq}}$ has the same units as the translational stiffnesses $\sigma_{i}$ and its equivalence is also based on the physically meaningful principle of elastic energy. The three parameters can now be meaningfully compared. We define the quality measure to be: $Q=\min \left\{\sigma_{1}, \sigma_{2}, \mu_{\mathrm{eq}}\right\}$, with higher $Q$-value implying a better grasp.

To define a quality measure for 3-D grasps, we similarly define stiffness parameters which are equivalent to the principal rotational stiffnesses $\mu_{i}(i=1,2,3)$. Let $\omega_{i}$ be a unit-magnitude eigenvector of $K_{V}$ associated with $\mu_{i}$, and let $\alpha$ be a scalar. Using formula (5) for $P$, the displacement of $\mathcal{B}$ represented by the vector $\alpha \omega_{i}$ is $\dot{q}_{i}=\alpha P \omega_{i}=\alpha\left(v_{i}, \omega_{i}\right)$, where $v_{i}=-K_{11}^{-1} K_{12} \omega_{i}$. When $\mathcal{B}$ is displaced by $\dot{q}_{i}$, the amount of induced elastic energy is $(1 / 2) \mu_{i} \alpha^{2}$, and $\mathcal{B}$ 's deflection is $\alpha\left(\rho_{\max _{i}}^{2}+\left(v_{i} \cdot \omega_{i}\right)^{2}\right)^{1 / 2}$, where $\rho_{\max _{i}}=\rho_{\max }\left(\dot{q}_{i}\right)$. Now imagine that the object, while attached to a linear spring, undergoes a pure translation by the amount of this deflection in the direction of the spring. We define the stiffness coefficient of the linear spring, denoted $\mu_{\mathrm{eq}_{i}}$, as equivalent to the principal rotational stiffness $\mu_{i}$, if the elastic energy of the spring due to the translation equals $(1 / 2) \mu_{i} \alpha^{2}$. Hence, by requiring that $(1 / 2) \mu_{\mathrm{eq}_{i}}\left(\alpha \sqrt{\rho_{\max _{i}}^{2}+\left(v_{i} \cdot \omega_{i}\right)^{2}}\right)^{2}=(1 / 2) \mu_{i} \alpha^{2}$, we obtain the following formula for $\mu_{\mathrm{eq}}$ :

$$
\mu_{\mathrm{eq}_{i}}=\frac{\mu_{i}}{\rho_{\max _{i}}^{2}+\left(v_{i} \cdot \omega_{i}\right)^{2}}, \quad \text { for } i=1,2,3 \text {. }
$$

As in the 2-D case, the equivalent stiffness parameters have the same units as the translational stiffnesses, and the quality measure is defined for 3-D grasps as follows. 
Definition 2: The stiffness quality measure for 2-D and 3-D compliant grasps is

$$
Q= \begin{cases}\min \left\{\sigma_{1}, \sigma_{2}, \mu_{\mathrm{eq}}\right\}, & \text { 2-D case } \\ \min \left\{\sigma_{\min }, \mu_{\mathrm{eq}}\right. & \text { 3-D case }\end{cases}
$$

where $\sigma_{\min }=\min \left\{\sigma_{1}, \sigma_{2}, \sigma_{3}\right\}$ and $\mu_{\mathrm{eq}_{\min }}=\min \left\{\mu_{\mathrm{eq}_{1}}, \mu_{\mathrm{eq}_{2}}\right.$, $\left.\mu_{\mathrm{eq}_{3}}\right\}$.

Since the principal stiffness parameters characterize the stiffness of a given grasp, the quality measure characterizes the grasp's worst-case stiffness. We note that $Q$ has the following properties. First, $Q$ is valid for grasps of 2-D and 3-D objects by any number of fingers. Second, the grasps can be modeled by any compliance model, since $Q$ depends only on the stiffness matrix of the grasp. Third, $Q$ is invariant with respect to change of world and object reference frames. Last, the optimal grasp of an object is the one which maximizes $Q$, since this grasp has the highest worst-case stiffness.

We conclude this section with an example, in which the stiffness quality measure is applied to the frictional grasp of a polygonal object, and is compared with the rigid grasp quality measure of Mirtich and Canny [13]. Examples of frictionless grasps are given in the next section. These examples will demonstrate that our grasp quality measure can be used to find better grasps when compliance is considered an important issue.

Example 1: Fig. 3(a) shows a regular pentagon whose edges are of length $a$. The pentagon is to be grasped by three frictional, compliant fingers. The figure depicts the following family of candidate grasps. In each grasp, a finger is located at the edge $C D$ 's midpoint, while the other two fingers lie on edges $A B$ and $A E$ at a distance $\lambda a$ from the vertex $A(0 \leqslant \lambda \leqslant 1)$. We wish to examine the variation of the stiffness quality measure as a function of $\lambda$. To compute the stiffness matrix of the frictional grasps, we use the following formula given in [3]:

$$
K=\sum_{i=1}^{3}\left(k_{n_{i}} \phi_{i} \phi_{i}^{T}+k_{t_{i}} \psi_{i} \psi_{i}^{T}\right) .
$$

In this formula, $n_{i}$ and $t_{i}$ are the unit normal and tangent vector at the $i$ th contact, and $k_{n_{i}}$ and $k_{t_{i}}$ are the material elasticity constants along $n_{i}$ and $t_{i}$. In addition, $\phi_{i}$ and $\psi_{i}$ are $3 \times 1$ vectors given by $\phi_{i}=\left(n_{i}, s_{n_{i}}\right), \psi_{i}=\left(t_{i}, s_{t_{i}}\right)$, where $s_{n_{i}}$ and $s_{t_{i}}$ are the respective moments of $n_{i}$ and $t_{i}$ with respect to the origin. Since the stiffness quality measure is frame-invariant, the world and body frames are conveniently chosen to be coincident, with origin lying at the pentagon's center of symmetry, and with $x$-axis parallel to the edge $C D$. Assuming for purposes of illustration that $k_{n_{i}}=k_{t_{i}}=k$, the stiffness matrix is

$$
K=k\left(\begin{array}{ccc}
3 & 0 & a(1.18 \lambda-1.01) \\
0 & 3 & 0 \\
a(1.18 \lambda-1.01) & 0 & a^{2}\left(2 \lambda^{2}-2 \lambda+1.92\right)
\end{array}\right)
$$

Thus, we can compute the quality measure as a function of $\lambda$, which is plotted in Fig. 3(b). It can be seen that as $\lambda$ increases within the interval $(0,0.86)$, the quality measure increases monotonically. On the other hand, for $0.86 \leqslant \lambda \leqslant 1$, the quality measure is nearly a constant, and achieves the maximum value $Q=2.34 k$ at $\lambda=1$.

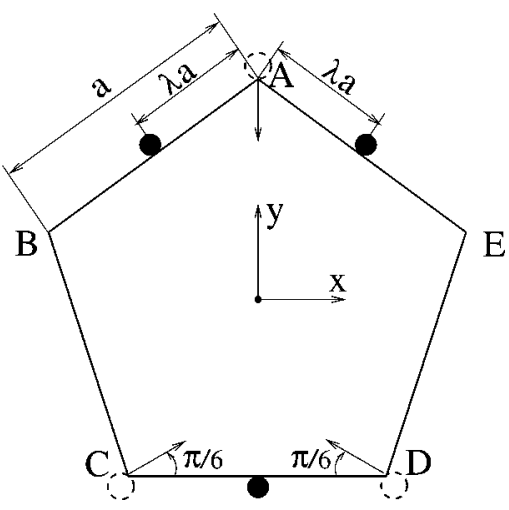

(a)

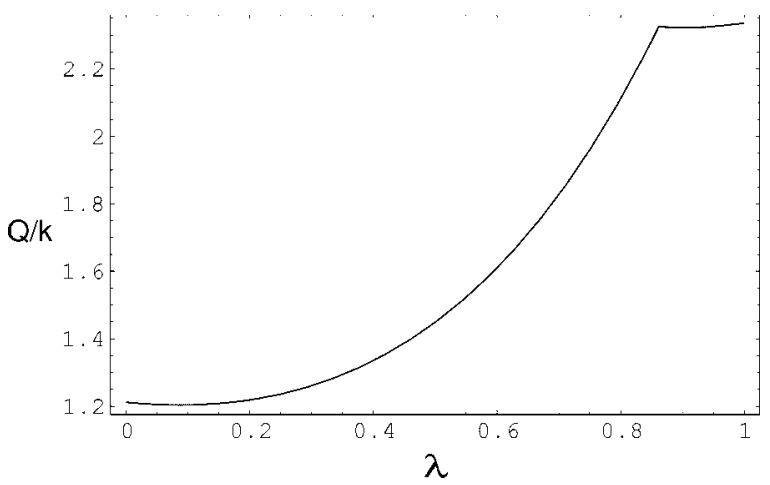

(b)

Fig. 3. (a) Three-finger frictional grasps of a regular pentagon. (b) Variation of the stiffness quality measure as a function of $\lambda$.

For purposes of comparison, let us apply the rigid-body grasp quality measure developed by Mirtich and Canny [13] to this grasp. The corresponding optimal rigid grasp places the fingers at the vertices $A, C$ and $D$, respectively, and each pair of the contact normals make an angle of $120^{\circ}$. The stiffness quality measure for this grasp is $Q=1.98 k$. Hence, the grasp determined by our quality measure has an $18 \%$ higher characteristic stiffness. In general, the optimal rigid-body grasp according to Mirtich and Canny [13] has the following two characteristics. Let $p$ be the concurrency point of the three finger forces. Then the fingers are evenly spread around $p$ for optimal worst-case force rejection, and the fingers are spread away from $p$ for optimal worst-case torque rejection. Our quality measure attempts to maximize the worst-case grasp stiffness, which consists of translational and equivalent rotational stiffnesses. As discussed below, the optimal translational stiffness leads to grasps whose fingers are evenly spread around $p$. However, the optimal rotational stiffness is determined by several simultaneous factors. These factors include the property that the fingers be spread away from $p$. Another factor is the property that the grasp's concurrency point be as close as possible to the object's geometrical center. ${ }^{4}$ This property has the effect of minimizing the worst-case deflection of the object points. Last, when applied to curved objects (not discussed here), the fingers tend to contact the object at points of low curvature, where the grasp's rotational stiffness is the highest.

\footnotetext{
${ }^{4}$ The geometric center of a planar object is the center of the object's smallest containing disc.
} 


\section{OPTIMAL GRASPING OF FRICTIONLESS POLYGONS}

The quality measure introduced above is valid for a large range of compliance and contact models. Here we consider in detail the application of the stiffness quality measure to the optimal grasping of frictionless polygonal objects by three and four fingers. In these cases we are able to obtain strong results. That is, these particular optimal grasps can be physically well characterized, easily computed, and in some cases found by inspection. In practice, these results are most suited for fixturing applications involving significant manufacturing forces, where it is not desirable to rely upon frictional forces at the contacts to achieve force-closure or stability [26].

To compute the stiffness matrix and hence the stiffness quality measure for such grasps, we employ the compliance model developed in [6] and [7]. This compliance model is compatible with the classical contact theory of Hertz [29], and we use this model under the assumption of linear elasticity at each contact. To further simplify the computation, we use disc fingers and allow the fingers to touch the object only along its edges. (However, it should be noted that the stiffness quality measure is valid for arbitrary fingers at any contact location and for any compliance model that yields a stiffness matrix.) In the cases discussed below we first characterize the $n$-finger stable equilibrium grasps, then discuss the optimization of $Q$ over these grasps.

\section{A. Optimal Three-Finger Grasps}

First we characterize the stable three-finger equilibrium grasps of a polygonal object. At a three-finger equilibrium grasp, the contact normals must positively span the origin such that their lines intersect at a common point. We discard degenerate three-finger equilibrium grasps where the three fingers touch only two edges of $\mathcal{B}$, since without friction these grasps are only neutrally stable. Thus, we focus on edge triplets which determine feasible nondegenerate grasps, and refer to these triplets as admissible. An admissible edge-triplets yields a stable equilibrium grasp when the stiffness matrix associated with the grasp is positive definite. The following lemma gives a formula for the stiffness matrix of a three-finger grasp of a polygon. In the lemma, $n_{i}$ is the inward unit normal to an edge of $\mathcal{B}$ at the $i$ th contact, and $k_{i}$ is the material elasticity at the $i$ th contact. Further, the circumscribing circle of a triangle is the circle which passes through the triangle's vertices (Fig. 4).

Lemma 3 [28]: Let three disc fingers hold a polygonal object $\mathcal{B}$ on an edge-triplet in a frictionless equilibrium grasp. Let the frames $\mathcal{F}_{W}$ and $\mathcal{F}_{B}$ be coincident at the concurrency point of the contact normal lines. Then the grasp stiffness matrix is given by

$$
K=\operatorname{diag}\left(\sum_{i=1}^{3} k_{i} n_{i} n_{i}^{T}, \mu\right), \quad \text { where } \mu=f_{T}(2 a \zeta+r) .
$$

In this formula, $f_{T}=\sum_{i=1}^{3} f_{i_{0}}$ where $f_{i_{0}}$ is the initial preloading force of the $i$ th finger the absence of an external wrench; $a$ is the radius of the triangle's circumscribing circle; $\zeta=\left(\prod_{i=1}^{3} \sin \alpha_{i}\right) /\left(\sum_{i=1}^{3} \sin \alpha_{i}\right)$ is determined from the

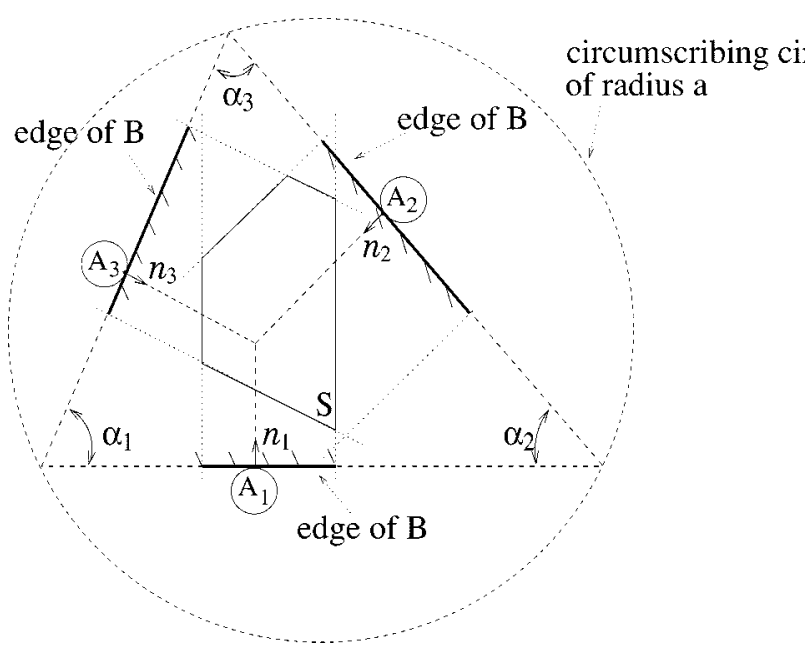

Fig. 4. Three fingers on an edge triplet.

triangle's three interior angles, denoted $\alpha_{i}(i=1,2,3)$; and $r$ is the radius of the disc fingers.

The lemma asserts that $K$ is block-diagonal when $\mathcal{F}_{B}$ 's origin is at the concurrency point of the contact normals, which is also the grasp's center of compliance. Thus, the eigenvalues of the $2 \times 2$ matrix $K_{11}=\sum_{i=1}^{3} k_{i} n_{i} n_{i}^{T}$ are the translational stiffnesses $\sigma_{1}, \sigma_{2}$, and $\mu$ is the rotational stiffness of the grasp. For $K$ to be positive definite, the three parameters must be positive. Since the submatrix $K_{11}$ is positive definite and constant on a given admissible edge-triplet, $\sigma_{1}$ and $\sigma_{2}$ are positive constants for all grasps associated with such an edge-triplet. In the rotational stiffness, $\mu=f_{T}(2 a \zeta+r), a$ and $r$ are positive constants, while $\zeta$ is a positive constant in compressive grasps where the fingers push toward the concurrency point. ${ }^{5}$ Assuming a compressive grasp, $\mu$ is positive when $f_{T}$ is strictly positive. Since $f_{T}$ is the sum of the preloading forces, the condition $f_{T}>0$ implies that the grasp must be preloaded for stability. We therefore assume that $f_{T}$ has a specified positive value for all possible finger placements. This is a reasonable assumption, since in practice one often wishes to compare different grasps having a common preloading level that is determined by task specifications and material strength limits. Thus $\mu$ is a positive constant on a given edge-triplet, and all preloaded equilibrium grasps on an admissible edge-triplet are stable.

Before proceeding with the stiffness analysis of three-finger grasps of polygons, we pause to discuss the effect of curvature on grasp stability. In [7], we compute the stiffness matrix of an $n$-finger grasp, in a way which is compatible with the Hertz contact theory. The computation is carried out for general bodies, and the curvature of the contacting bodies plays an important role in the grasp's stability. A discussion of the role of curvature in grasp stability appears in [7] and can be roughly summarized as follows. When a finger and an object are strictly convex at their contact point, an increase of the contact force at that point destabilizes the grasp. Conversely, when one of the contacting bodies is either flat or concave, an increase of the contact force stabilizes the grasp. These findings are consistent with the "coin

\footnotetext{
${ }^{5} \zeta$ is negative in expansive grasps where the fingers push away from the concurrency point
} 
snapping" problem mentioned in [5]. These findings are also consistent with the stiffness matrix formula (10), which asserts that when disc fingers contact polygonal edges, an increase of the preloading forces enhances the grasp's stability.

We restrict our attention to nondegenerate edge triplets, whose edges are not nearly parallel. (Clearly, finger placement on degenerate edge triplets is generally not desirable in practice.) We now show that for nondegenerate edge triplets, $\mu_{\text {eq }} \leqslant \sigma_{1}, \sigma_{2}$. First, it directly follows from the structure of $K_{11}$ that $\sigma_{1}$ and $\sigma_{2}$ are on the same order as $k_{i}$. On the other hand, let $l$ be the object's characteristic dimension. Then $\mu=f_{T}(2 a \zeta+r)$ is on the same order as $f_{T} \cdot l$. Since $\rho_{\max }$ is also on the order of $l, \mu_{\mathrm{eq}}=\mu / \rho_{\max }^{2}$ is on the order of $f_{T} / l$. However, it can be verified that for small deformations at the contacts, $f_{T} / l \ll k_{i}$ [28]. Hence, $\mu_{\mathrm{eq}}$ is much smaller than $\sigma_{1}$ and $\sigma_{2}$, and we obtain

$$
Q=\min \left\{\sigma_{1}, \sigma_{2}, \mu_{\mathrm{eq}}\right\}=\mu_{\mathrm{eq}}=f_{T} \frac{2 a \zeta+r}{\rho_{\max }^{2}} .
$$

In words, three-finger grasps are not highly "rotationally stiff" about the concurrency point, and for such grasps $Q$ is dominated by the equivalent rotational stiffness. Note that under a pure rigid-body model, four contacts are required for frictionless form-closure and hence stable grasp. However, here curvature effects are taken into account, and three compliant contacts suffice to generate a positive definite stiffness matrix and hence a stable grasp. See [6] for a discussion of curvature and its effect on grasp stability.

In (11), the numerator is constant for all grasps on a given edge-triplet. Hence, $Q$ is maximized when $\rho_{\max }^{2}$ is minimized. This agrees with our intuition, since the grasp with the smallest $\rho_{\max }$ has the largest equivalent rotational stiffness about its concurrency point. To compute the grasp which minimizes $\rho_{\max }^{2}$ on a given edge-triplet, we parametrize the equilibrium grasps on the edge-triplet by the coordinates of the concurrency point, denoted $p$. The domain of $p$ is a convex polygonal region $S$, which is formed by intersecting three strips orthogonal to the edges (Fig. 4). For each $p \in S$ there exist finger placements such that $p$ is the concurrency point of the contact normals. Let $v_{1}, \ldots, v_{n}$ be the vertices of $\mathcal{B}$. Then $\rho_{\max }^{2}(p)=\max \left\{\| v_{i}-\right.$ $\left.p \|^{2}\right\}$ over the vertices $v_{1}, \ldots, v_{n}$. It can be verified the $\rho_{\max }^{2}(p)$ is a convex function. Since the region $S$ is convex, the minimization of $\rho_{\max }^{2}(p)$ over $S$ is a standard convex minimization problem, which can be solved by efficient $\epsilon$-approximate algorithms in $O(n \log (1 / \epsilon))$ steps [30]. [The coefficient $\log (1 / \epsilon)$ grows rather slowly with the desired accuracy $\epsilon$, and is effectively a constant.] The globally optimal grasp can be found by computing the optimal $Q$-value over all admissible edge-triplets of $\mathcal{B}$. According to [31], the collection of all feasible edgetriplets can be computed in $O\left(n^{2} \log ^{2} n+M\right)$ steps, where $M$ is the actual number of feasible edge-triplets and is $O\left(n^{3}\right)$ in worst case. Neglecting the $\log (1 / \epsilon)$ coefficient, the globally optimal three-finger grasp of a polygon can be computed in $O\left(n^{3} \log ^{2} n+M n\right)$ steps.

Inspection of formula (11) reveals the following qualitative characteristics of the globally optimal grasp. In (11), while the total preloading $f_{T}$ is taken to be the same for all edge triplets, the quantities $a$ and $\zeta$ are different for different edge triplets.

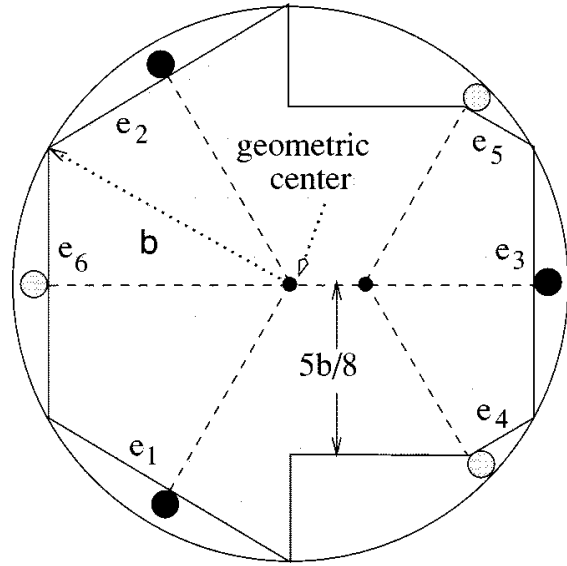

Fig. 5. Optimal three-finger grasps of a polygon along the edge triplets $\left(e_{1}, e_{2}, e_{3}\right)$ and $\left(e_{4}, e_{5}, e_{6}\right)$. The globally optimal grasp is indicated by solid circles.

Therefore, whether the optimal grasp on a given edge-triplet is the global optimum over all edge-triplets depends on the distance $\rho_{\max }$, as well as the shape (characterized by $\zeta$ ) and the size (characterized by $a$ ) of the triangle determined by the given edge triplet. For the quality measure to assume a large value, $\rho_{\max }$ is preferred to be small, while $a$ and $\zeta$ are preferred to be large. It can be verified that the shape parameter $\zeta$ is bounded by $\zeta \leqslant 1 / 4$ [28], with equality holding for an equilateral triangle. Thus, the edges in the triplet are preferred to be evenly oriented. In particular, for uniform material constants $k_{i}=k$, the optimum contact normals are $120^{\circ}$ apart. Moreover, since the parameter $a$ is preferred to be large, the contacts are preferred to be spread apart. We illustrate these observations with the following example.

Example 2: Consider the grasping of a polygonal object by three fingers, which are assumed to have zero radius for simplicity. As shown in Fig. 5, the polygon's convex hull is a regular hexagon. The polygon's geometric center (i.e., the center of the object's smallest containing disc) is given by the center of the hexagon's circumscribing circle. We consider two edge-triplets $\left(e_{1}, e_{2}, e_{3}\right)$ and $\left(e_{4}, e_{5}, e_{6}\right)$. These two edge-triplets determine two congruent triangles, for which the combined effect on $Q$ of shape and size is given by $a \zeta=(\sqrt{3} / 4) b$. The optimal concurrency point of the triplet $\left(e_{1}, e_{2}, e_{3}\right)$ coincides with the geometric center. Thus, $\rho_{\max }=b$ and $Q=(\sqrt{3} / 2)\left(f_{T} / b\right)$ for this grasp. On the other hand, the optimal concurrency point of $\left(e_{3}, e_{4}, e_{5}\right)$ lies on the line of symmetry of $\mathcal{B}$, at a distance $(\sqrt{3} / 6) b$ from the geometric center. For this finger arrangement, it can be shown that $\rho_{\max }^{2}=(19 / 12) b^{2}$ and $Q=(6 \sqrt{3} / 19)\left(f_{T} / b\right)$. We see that while the triangles associated with the two edge-triplets have the same shape and size, the different distance of the optimal concurrency point from the farthest vertices of $\mathcal{B}$ lead to different quality measure values. Consequently, the optimal grasp on $\left(e_{1}, e_{2}, e_{3}\right)$ is better than the optimal grasp on $\left(e_{4}, e_{5}, e_{6}\right)$.

Finally, the following example illustrates that it is possible to find the globally optimal grasp of simple objects graphically.

Example 3: Let $\mathcal{B}$ be a triangular object, and let $p_{0}$ be the center of the smallest disc containing the object. As shown in 


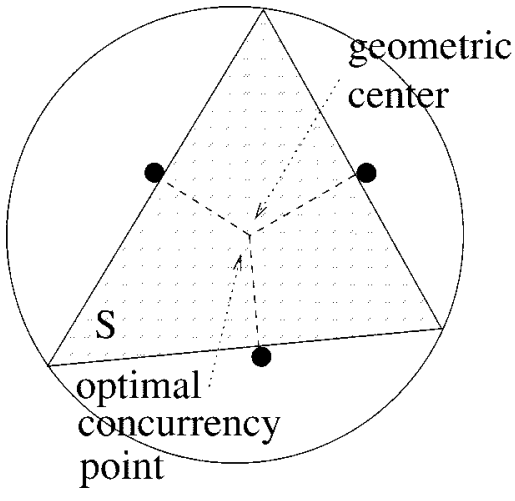

(a)

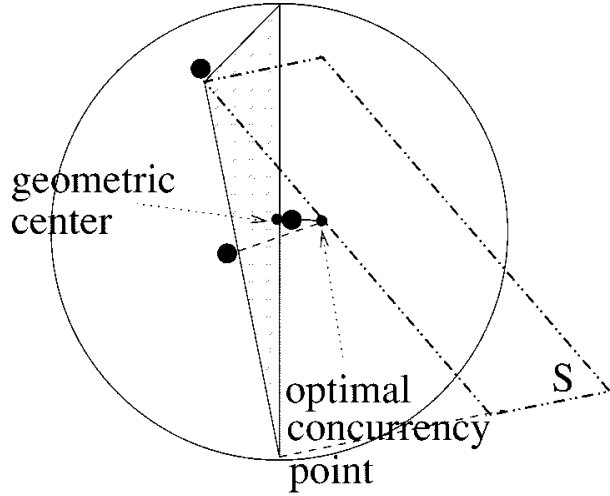

(b)

Fig. 6. Optimal three-finger grasps of two triangular objects, in which $p_{0}$ lies (a) inside $S$ and (b) outside $S$.

Fig. 6(a), if the region $S$ contains the point $p_{0}$, the optimal concurrency point is at $p_{0}$. Otherwise, $p_{0}$ lies outside $S$. In this case $\mathcal{B}$ must be an obtuse triangle, and $p_{0}$ lies at the midpoint of the triangle's longest edge [Fig. 6(b)]. The region $S$ is then a parallelogram whose geometry is determined by the other two edges of the triangle. The optimal concurrency point is at the center of the smallest disc which contains $\mathcal{B}$, such that this center lies in $S$. To find this point, construct the half-line that simultaneously passes through $p_{0}$ and is perpendicular to the longest edge of $\mathcal{B}$. Then the point where this line first intersects the region $S$ is the optimal concurrency point, see Fig. 6(b).

\section{B. Optimal Four-Finger Grasps}

We now consider the extension of the above results to four frictionless contacts. Stable four-finger grasps of a frictionless polygonal object involve either three or four edges. Hence, we must consider all four-finger placements on triplets and quadruples of edges. The four-finger grasps on a given edge combination are parametrized as follows. Let $O$ be the origin of $\mathcal{F}_{B}$ and let $e_{i}$ be the edge containing the $i$ th contact. The $i$ th contact is parametrized by the signed distance $s_{i}$ along $e_{i}$ of the $i$ th contact from the point where $e_{i}$ (or its extension) perpendicularly intersects a line passing through $O$. (The parameter $s_{i}$ is the torque generated by a unit force $n_{i}$ acting on $\mathcal{B}$ at the point $r_{i}$ ). A point $s=\left(s_{1}, s_{2}, s_{3}, s_{4}\right)$ specifies a particular four-finger grasp, and the collection of all four-finger grasps on a given edge combination is a rectangular parallelepiped $\mathcal{P}$ in $\mathbb{R}^{4}$.

To characterize the four-finger stable equilibrium grasps in $\mathcal{P}$, let $\boldsymbol{w}_{i}=\left(n_{i}, s_{i}\right)$ denote the wrench generated by a unit force $n_{i}$ acting on $\mathcal{B}$ at the $i$ th contact. Then at a four-finger equilibrium grasp a positive linear combination of these wrenches must give the zero wrench. This equilibrium condition is valid on a subset of $\mathcal{P}$ which is characterized as follows. Let the functions $d_{i}(s)=\operatorname{det}\left(\left[\boldsymbol{w}_{i+1} \boldsymbol{w}_{i+2} \boldsymbol{w}_{i+3}\right]\right)(\bmod 4)$ be called the $d e-$ terminant functions associated with the given edge combination. Then we have the following lemma.

Lemma 4 [28]: A necessary and sufficient condition for a four-finger grasp with a contact configuration $s \in \mathcal{P}$ to be an equilibrium grasp is that $d_{1}(s),-d_{2}(s), d_{3}(s)$, and $-d_{4}(s)$ are all nonzero and have the same sign.
Thus, the collection of equilibrium grasps is the union $\mathcal{T}=$ $\mathcal{T}_{1} \cup \mathcal{T}_{2}$, where

$$
\begin{aligned}
& \mathcal{T}_{1}=\mathcal{P} \cap\left\{s \in \mathbb{R}^{4}: d_{1}(s),-d_{2}(s), d_{3}(s),-d_{4}(s)<0\right\} \\
& \mathcal{T}_{2}=\mathcal{P} \cap\left\{s \in \mathbb{R}^{4}: d_{1}(s),-d_{2}(s), d_{3}(s),-d_{4}(s)>0\right\}
\end{aligned}
$$

Since each function $d_{i}$ is linear in $s$, each $\mathcal{T}_{i}$ is a bounded convex polytope in $\mathbb{R}^{4}$. Thus, for a given edge combination we may separately search the convex polytopes $\mathcal{T}_{1}$ and $\mathcal{T}_{2}$ for the optimal finger arrangement. The following lemma gives a formula for the stiffness matrix of a four-finger grasp of a polygon.

Lemma 5 [28]: Let four fingers hold a polygonal object $\mathcal{B}$ on four edges in a frictionless equilibrium grasp. Let the frames $\mathcal{F}_{W}$ and $\mathcal{F}_{B}$ be coincident. Then the grasp stiffness matrix is given by

$$
K=\sum_{i=1}^{4} k_{i} \boldsymbol{w}_{i} w_{i}^{T}=\left(\begin{array}{cc}
\sum_{i=1}^{4} k_{i} n_{i} n_{i}^{T} & \sum_{i=1}^{4} k_{i} s_{i} n_{i} \\
\sum_{i=1}^{4} k_{i} s_{i} n_{i}^{T} & \sum_{i=1}^{4} k_{i} s_{i}^{2}
\end{array}\right)
$$

where $k_{i}$ is the material elasticity constant at the $i$ th contact, and $n_{i}$ the inward unit normal at the $i$ th contact.

Note that $K=H H^{T}$ where $H=\left[\sqrt{k_{1}} \boldsymbol{w}_{1} \cdots \sqrt{k_{4}} \boldsymbol{w}_{4}\right]_{3 \times 4}$. Hence, $K$ is positive-definite when $H$ has full rank, which holds true for all generic four-finger equilibrium grasps. Thus all generic four-finger equilibrium grasps are stable. Note, as well, that the particular shape of the fingers does not appear in the stiffness matrix formula. This is in contrast with the three-finger stiffness matrix formula, in which the fingers' radius $r$ appears in the grasp's rotational stiffness term. In fact, formula (12) is a simplification of an exact formula which contains an additional curvature-dependent term [7]. However, this term is typically much smaller than the first-order term appearing in (12), and it does not affect the grasp stability.

Next, we derive a formula for $Q$ on a particular edge combination. Recall that the translational stiffness parameters are the eigenvalues of the submatrix $K_{11}$, while the rotational stiffness parameter is given by $\mu=K_{22}-K_{12}^{T} K_{11}^{-1} K_{12}$. Using (12), the submatrix $K_{11}=\sum_{i=1}^{4} k_{i} n_{i} n_{i}^{T}$ is constant for all grasps on 
a given edge combination. Hence, $\sigma_{1}, \sigma_{2}$ are positive constants for all grasps on a given edge combination. As for $\mu$, substitution of the submatrices $K_{i j}$ according to (12) gives

$$
\begin{aligned}
& \mu(s)=\sum_{i=1}^{4} k_{i} s_{i}^{2}-\left(\sum_{i=1}^{4} k_{i} s_{i} n_{i}^{T}\right) \\
& \cdot\left(\sum_{i=1}^{4} k_{i} n_{i} n_{i}^{T}\right)^{-1} \sum_{i=1}^{4} k_{i} s_{i} n_{i}
\end{aligned}
$$

which can be shown to be a nonnegative quadratic function of $s$. To compute $Q$, we also need a formula for the equivalent rotational stiffness $\mu_{\mathrm{eq}}=\mu / \rho_{\max }^{2}$, where $\rho_{\max }$ is the distance from the grasp's center of compliance to the farthest vertex of $\mathcal{B}$. Let $p$ denote the grasp's center of compliance. Then according to (7), $p=J K_{11}^{-1} K_{12}$, where we assume that the frame $\mathcal{F}_{B}$ is aligned with the frame $\mathcal{F}_{W}$. Substituting for $K_{i j}$ according to Lemma 5 gives: $p(s)=J\left[\sum_{i=1}^{4} k_{i} n_{i} n_{i}^{T}\right]^{-1} \sum_{i=1}^{4} k_{i} s_{i} n_{i}$, which is linear in $s$. Thus, $\rho_{\max }^{2}(p(s))=\max \left\{\left\|v_{i}-p(s)\right\|^{2}\right\}$ over the vertices $v_{1}, \ldots, v_{n}$ of $\mathcal{B}$. Since $p(s)$ is linear in $s, \rho_{\max }^{2}(p(s))$ is the maximum of $n$ positive-definite quadratic functions in $s$. Thus, the maximum value of $Q$ on a particular edge combination is

$$
Q=\min \left\{\sigma_{1}, \sigma_{2}, \max _{s \in \mathcal{T}_{1} \cup \mathcal{T}_{2}}\left\{\frac{\mu(s)}{\rho_{\max }^{2}(p(s))}\right\}\right\} .
$$

In the Appendix, we present a procedure for finding the global solution of the maximization problem $\max _{s \in \mathcal{T}_{i}}\left\{\mu(s) / \rho_{\max }^{2}(p(s))\right\}$ in terms of $n$ indefinite quadratic programming (IQP) problems. While IQP is NP-hard, there exist approximation algorithms which are polynomial in the number of constraints. Finally, we have to perform an IQP optimization on all edge triplets and quadruples associated with feasible four-finger grasps. According to [31], these edge combinations can be computed in $O\left(n^{2}+M\right)$ steps, where $M$ is the actual number of feasible edge-combinations and is $O\left(n^{4}\right)$ in worst case.

Before presenting some examples, we mention several characteristics of the optimal four-finger grasp on a given edge combination. First, $\sigma_{\min }=\min \left\{\sigma_{1}, \sigma_{2}\right\}$ is constant for all grasps on a given edge combination. Moreover, the formula $Q=\min \left\{\sigma_{1}, \sigma_{2}, \mu_{\mathrm{eq}}\right\}$ indicates that $Q \leqslant \sigma_{\min }$ on a given edge combination. Hence, if we find in the course of maximizing $\mu_{\mathrm{eq}}(s)$ some $s^{*}$ such that $\mu_{\mathrm{eq}}\left(s^{*}\right) \geqslant \sigma_{\min }$, this $s^{*}$ is necessarily the optimal finger arrangement on the given edge combination. Second, $\sigma_{\min }$ is the smallest eigenvalue of the submatrix $K_{11}=\sum_{i=1}^{4} k_{i} n_{i} n_{i}^{T}$, denoted $\lambda_{\min }\left(K_{11}\right)$. Hence, $Q \leqslant \lambda_{\min }\left(K_{11}\right)$. In order to achieve the highest value of $\lambda_{\min }\left(K_{11}\right)$ among all edge combinations, the contact normals $n_{i}$ are preferred to be evenly oriented. In particular, for uniform material constants $k_{i}=k$, the optimum contact normals are $90^{\circ}$ apart. Next, we discuss the parameters that influence $\mu_{\mathrm{eq}}(s)$. Since $\mu_{\mathrm{eq}}(s)=\mu(s) / \rho_{\max }^{2}(p(s))$, the parameter $\rho_{\max }$ is desired to be small while $\mu$ is preferred to be large. If we choose the frames $\mathcal{F}_{W}$ and $\mathcal{F}_{B}$ at the grasp's center of compliance, the stiffness matrix in (12) becomes diagonal. In this case $\mu=\sum_{i=1}^{4} k_{i} \bar{s}_{i}^{2}$, where $\bar{s}_{i}$ is the $i$ th contact's moment about the center of compliance. Thus, for $\mu$ to assume a large value, each $\left|\bar{s}_{i}\right|$ is desired to be large. This indicates that the

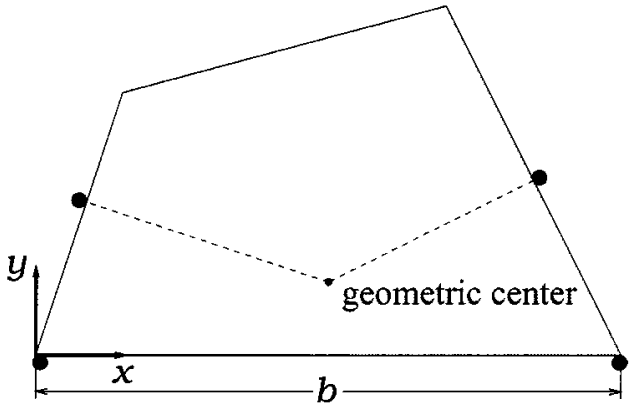

Fig. 7. Four-finger grasp of a general quadrilateral object. The optimal grasp is indicated by solid circles.

fingers should spread apart as much as possible with respect to the grasp's center of compliance. To summarize, for a stiff grasp, the edges should be evenly oriented; the fingers should spread apart with respect to the center of compliance; and the distance from the grasp's center of compliance to $\mathcal{B}^{\prime}$ farthest vertex should be small.

In the following examples, we assume point fingers and uniform material elasticity constants of $k_{i}=k$.

Example 4: Fig. 7 shows a general quadrilateral object grasped by four frictionless fingers. For such a general polygonal object, it is necessary to use the numerical procedure outlined in the Appendix. The quadrilateral's vertices have coordinates $(0,0),(b, 0),(0.7 b, 0.6 b)$ and $(0.15 b, 0.45 b)$, and its geometric center is located at $(0.5 b, 0.125 b)$. After considering all feasible edge combinations, the globally optimal grasp is shown in Fig. 7. For this grasp, $\sigma_{\min }=1.684 k$ and $\mu_{\mathrm{eq}}=1.882 k$, hence $Q=1.684 k$. In addition, the grasp's center of compliance coincides with the object's geometric center. Since $\mu_{\mathrm{eq}}=\mu / \rho_{\max }^{2}$, the optimal finger arrangement maximizes $\mu_{\mathrm{eq}}$ by minimizing $\rho_{\max }$, and by spreading apart the two fingers on the base edge to allow $\mu$ to assume a large value.

Finally, objects with special symmetry allow graphical analysis of the optimal finger arrangement. The following two examples consider two such objects.

Example 5: Fig. 8(a) shows a rectangle of size $2 a \times 2 b$ grasped by four frictionless fingers. In any equilibrium grasp of the rectangle, the lines of the contact normals form a rectangle, which we call the grasp rectangle. Since each finger is located on a different edge of $\mathcal{B}$, the contact normals are oriented $90^{\circ}$ apart. This, it can be shown, implies that $\sigma_{\min }=2 k$ for all finger arrangements. As for the rotational stiffness $\mu$, it can be verified that $\mu=2 k\left(\bar{s}_{1}^{2}+\bar{s}_{2}^{2}\right)$, where $\bar{s}_{1}$ and $\bar{s}_{2}$ are the half-length and half-width of the grasp rectangle. Clearly, when the fingers are placed at the ends of the object's edges with $\bar{s}_{1}=a$ and $\bar{s}_{2}=b$, as shown with solid circles in the figure, $\mu$ achieves its maximum value: $\mu=2 k\left(a^{2}+b^{2}\right)$. Since $\mu_{\mathrm{eq}}=\mu / \rho_{\max }^{2}$, the same finger placement also maximizes the equivalent rotational stiffness: $\mu_{\mathrm{eq}}=2 k$. It follows that the finger arrangement shown with solid circles is the globally optimal grasp, with maximal value of $Q=2 k$.

Example 6: Fig. 8(b) shows an equilateral triangle grasped by four frictionless fingers. Without loss of generality, we focus on the placement of two fingers on the base edge, and one finger on each side edge. Using symmetry arguments [28], it can be shown that in the optimal grasp, the side fingers' force lines 


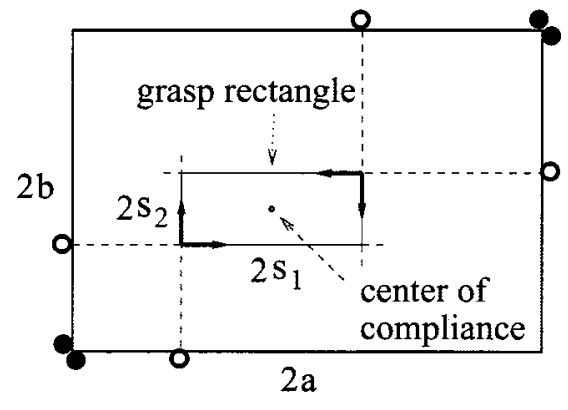

(a)

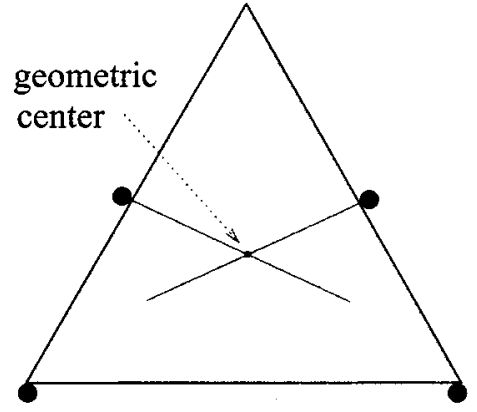

(b)

Fig. 8. Four-finger grasps of (a) a rectangle and (b) an equilateral triangle. The optimal grasps are indicated by solid circles.

intersect at $\mathcal{B}$ 's center of symmetry, while at the bottom the two fingers are located on opposite endpoints of the edge.

\section{CONCLUSION}

We described the frame-invariant parameters of the stiffness matrix and used these parameters to define a stiffness quality measure for compliant grasps or fixtures. The quality measure is based on the grasp's principal translational and principal rotational stiffnesses. In order to meaningfully compare rotational stiffnesses with translational stiffnesses, we introduced a notion of deflection and its related elastic energy. The resulting quality measure reflects the worst-case stiffness of a grasp. Generally, the higher the quality measure, the better the grasp. We also considered the computation of the optimal grasp of a frictionless polygonal object by three and four fingers. In each case, we also characterized the qualitative properties of the optimal grasp.

The stiffness quality measure has several important properties. First, the measure is independent of the choice of object and world frames. Second, the measure is explicitly designed for compliant grasps, and is the first systematic effort to quantify the effectiveness of compliant grasps. Moreover, the quality measure can be used by any compliance model. Third, the quality measure is valid for grasps of 2-D and 3-D objects by any number of fingers. In particular, it is known that curvature effects can significantly reduce the number of frictionless fingers or fixtures required to stably grasp an object [32]. If desired, the stiffness quality measure can automatically include first-order effects (i.e., finger positions and contact normal directions) with second-order effects (i.e., surface curvature at the contacts) in a single measure. The quality measure is thus useful for assessing in a uniform way the effectiveness of grasps which involve different number of fingers and different types of geometrical effects.

There are several issues that call for further research. First we mention two computational issues. While we developed an efficient procedure for computing the globally optimal planar grasp in the case of a frictionless linear compliance contact model, practical global optimization with nonlinear and frictional contact models needs to be addressed. Furthermore, while our quality measure can be used to evaluate the effectiveness of any given 3-D grasp, the computation of optimal 3-D grasps may be a nontrivial issue. Next, we mention two possibly better stiffness-based quality measures. The first is the generalization of our quality measure to grasps subjected to task-specific external wrenches, rather than arbitrary external wrenches. The second is a quality measure that estimates the object's maximal deflection under external wrenches. Here we used the notion of object deflection only to compare translational and rotational stiffnesses. However, the stiffness quality measure is not an indicator that directly assesses the object's maximal deflection, which may be desirable in applications such as workpiece fixturing. Preliminary results in this direction can be found in [33].

Finally we mention potential applications of this work. The stiffness quality measure is most useful for passive grasp and fixture planning. An important application of this type is workpiece fixturing, where a part is held by fixture elements for machining purposes. The fixture elements have to protect the workpiece from deflecting under the load of the machining forces, and the stiffness quality measure can indicate the number and location of fixture elements that best suit the given task. The quality measure is also useful for active grasp planning, where finger linkages have to stably grasp an object. As several researchers have suggested [1], [2], [] we may perform such tasks by simulating virtual springs at the contacts. The stiffness quality measure is useful for selecting the optimal placement and stiffness of such springs, based on the object's geometry. The measure also allows an inclusion of the fingers' geometry into the planning, thereby providing a tool for selecting among several possible finger geometries in applications where such a choice is available.

\section{APPENDIX \\ PROCEDURE FOR FINDING OPTIMAL FOUR-FINGER GRASPS OF A POLYGON}

In Section V-B, we showed that the four-finger optimal grasping problem requires a solution to the following problem:

$$
\max _{s \in \mathcal{D}} \mu_{\mathrm{eq}}(s)=\frac{\mu(s)}{f(s)}
$$

where $\mu$ and $f$ are positive semi-definite quadratic functions with $f(s)>0$ whenever $s \in \mathcal{D}$, and the domain $\mathcal{D}$ is a convex polyhedral subset of $\mathbb{R}^{4}$. We are interested in finding the global maximum of $\mu_{\mathrm{eq}}(s)$ over $\mathcal{D}$. However, $\mu_{\mathrm{eq}}$ is a nonconvex, strongly nonlinear function. In general, the global maximum for such problems may be very difficult to find. Fortunately, 
there exists an effective algorithm to find the global optimum in this case. First define a function $\phi: \mathbb{R} \times \mathcal{D} \rightarrow \mathbb{R}$ by

$$
\phi(t, s)=\mu(s)-t f(s)
$$

For a given $t \in \mathbb{R}$, there exists $s \in \mathcal{D}$ such that $t=\mu_{\mathrm{eq}}(s)$ if and only if $\phi(t, s)=0$. Thus, the maximization problem (13) is equivalent to maximizing $t \in \mathbb{R}$ such that $(t, s)$ is a zero of $\phi$ for some $s \in \mathcal{D}$. To address this equivalent problem, we further define the scalar function $\psi: \mathbb{R} \rightarrow \mathbb{R}$ as

$$
\psi(t)=\max _{s \in \mathcal{D}} \phi(t, s)
$$

For $\Delta t>0$, since $f(s)$ is strictly positive, we have

$$
\begin{aligned}
\psi(t+\Delta t) & =\max _{s \in \mathcal{D}}(\mu(s)-t f(s)-\Delta f(s)) \\
& <\max _{s \in \mathcal{D}}(\mu(s)-t f(s))=\psi(t) .
\end{aligned}
$$

In other words, $\psi$ is strictly monotonic decreasing. In addition, since $\psi(0)=\max _{s \in \mathcal{D}} \mu(s)>0$, and $\psi(t) \rightarrow-\infty$ as $t \rightarrow \infty$, there exists a unique $t^{*}>0$ such that $\psi\left(t^{*}\right)=0$. That is, $\psi$, has a unique, positive zero. The following proposition shows how to maximize $\mu_{\mathrm{eq}}(s)$ over $\mathcal{D}$.

Proposition 6: Maximizing $\mu_{\mathrm{eq}}(s)$ over $\mathcal{D}$ is equivalent to finding the unique zero of $\psi$ in the following sense. A positive number $t^{*}>0$ satisfies $\psi\left(t^{*}\right)=0$ if and only if $t^{*}=$ $\max _{s \in \mathcal{D}} \mu_{\mathrm{eq}}(s)$. In this case, a contact configuration $s^{*} \in \mathcal{D}$ maximizes $\phi\left(t^{*}, s\right)$, regarded as a function of $s$, over $\mathcal{D}$ if and only if it maximizes $\mu_{\mathrm{eq}}(s)$ over $\mathcal{D}$.

Proof: If $t^{*}=\mu_{\mathrm{eq}}\left(s^{*}\right)=\max _{s \in \mathcal{D}} \mu_{\mathrm{eq}}(s)$, then $\phi\left(t^{*}, s^{*}\right)=0$. For any $s \in \mathcal{D}$, we have

$$
\begin{aligned}
\phi\left(t^{*}, s\right) & =\mu(s)-t^{*} f(s) \\
& =f(s)\left(\mu_{\mathrm{eq}}(s)-t^{*}\right) \leqslant 0=\phi\left(t^{*}, s^{*}\right) .
\end{aligned}
$$

Hence, $\psi\left(t^{*}\right)=\phi\left(t^{*}, s^{*}\right)=\max _{s \in \mathcal{D}} \phi\left(t^{*}, s\right)=0$. Conversely, suppose that $\psi\left(t^{*}\right)=0$ for some $t^{*}>0$. Given any $\Delta t>0$, we have

$$
\phi\left(t^{*}+\Delta t, s\right) \leqslant \psi\left(t^{*}+\Delta t\right)<\psi\left(t^{*}\right)=0
$$

where the strict monotonicity of $\psi$ has been used. This indicates that there exist no $s \in \mathcal{D}$ such that $\phi\left(t^{*}+\Delta t, s\right)=0$ for any $\Delta t>0$. Hence, $t^{*}=\max _{s \in \mathcal{D}} \mu_{\mathrm{eq}}(s)$. Moreover, let $s^{*} \in \mathcal{D}$ be such that $\psi\left(t^{*}\right)=\phi\left(t^{*}, s^{*}\right)=\max _{s \in \mathcal{D}} \phi\left(t^{*}, s\right)$. Then by definition of $\phi$, we have $t^{*}=\mu\left(s^{*}\right) / f\left(s^{*}\right)=\mu_{\mathrm{eq}}\left(s^{*}\right)$. Hence, $s^{*}$ maximizes $\mu_{\mathrm{eq}}$.

This proposition states that the optimization problem (13) is equivalent to solving for the unique root of the equation $\psi(t)=0$. In order to evaluate $\psi$ at some $t$, which is required by the algorithm, we need to maximize a quadratic function of $s$, which is in general indefinite, i.e., the matrix associated with the quadratic term in this function has positive and negative eigenvalues. Indefinite quadratic programming (IQP) is unfortunately NP-hard, and known algorithms for exact solutions are exponential in the number of variables. References [34] and [35] describe an approximate IQP minimization algorithm which takes $O\left(l((m(m+1)) / \sqrt{\epsilon})^{p}\right)$ steps, where $m$ is the number of variables and $p$ the number of negative eigenvalues of the quadratic objective function. In this bound, $l$ is the time it takes to solve a convex quadratic optimization problem of the same size, which is $O(n \log \epsilon)$ where $n$ is the number of linear constraints in the polytope $\mathcal{D}$. The variable $\epsilon$ describes the degree of approximation. Hence, the number of steps is linear in the number of constraints. With $m$ being small, our approach provides a practical procedure which guarantees that the global optimum will be found at a reasonable computational cost despite the strongly nonlinear and nonconvex nature of the problem.

\section{REFERENCES}

[1] H. Hanafusa and H. Asada, "Stable prehension by a robot hand with elastic fingers," in Proc. 7th Int. Symp. Industrial Robots, 1977, pp. 361-368.

[2] V.-D. Nguyen, "Constructing stable grasps," Int. J. Robot. Res., vol. 8, no. 1 , pp. 26-37, 1989.

[3] J. P. Donoghue, W. S. Howard, and V. Kumar, "Stable workpiece fixturing," in Proc. ASME Design Technical Conf., 1994.

[4] W. S. Howard and V. Kumar, "Stability of planar grasps," in Proc. Int. Conf. Robotics and Automation, 1994, pp. 2822-2827.

[5] M. R. Cutkosky and P. Wright, "Friction, stability and the design of robotic fingers," Int. J. Robot. Res., vol. 5, no. 4, pp. 20-37, 1986.

[6] E. Rimon and J. W. Burdick, "Mobility of bodies in contact-Part ii: How forces are generated by curvature effects," IEEE Trans. Robot. Automat., vol. 14, pp. 709-717, Oct. 1998.

[7] Q. Lin, J. W. Burdick, and E. Rimon, "Computation and analysis of compliance in grasping and fixturing," in Proc. Int. Conf. Robotics and Automation, 1997, pp. 93-99.

[8] B. Mishra, "Grasp metrics: Optimality and complexity," in Proc. 1994 Workshop on Algorithmic Foundations of Robotics (WAFR), San Francisco, CA, Feb. 1994, pp. 137-165.

[9] Z. Li and S. Sastry, "Task oriented optimal grasping by multifingered robot hands," IEEE Trans. Robot. Automat., vol. 4, pp. 32-44, Feb. 1988.

[10] D. G. Kirkpatrick, B. Mishra, and C. K. Yap, "Quantitative Steinitz's theorems with applications to multifingered grasping," in Proc. 20th ACM Symp. Theory of Computing, Baltimore, MD, May 1990, pp. 341-351.

[11] C. Ferrari and J. Canny, "Planning optimal grasps," in Proc. Int. Conf. Robotics and Automation, Nice, France, May 1992, pp. 2290-2295.

[12] X. Markenscoff and C. H. Papadimitriou, "Optimum grip of a polygon," Int. J. Robot. Res., vol. 8, no. 2, pp. 17-29, 1989.

[13] B. Mirtich and J. Canny, "Easily computable optimum grasps in 2-D and 3-D," in Proc. Int. Conf. Robotics and Automation, 1994, pp. 739-747.

[14] M. Teichmann, "A grasp metric invariant under rigid motions," in Proc. Int. Conf. Robotics and Automation, 1996, pp. 2143-2148.

[15] Z. Ji and B. Roth, "Direct computation of grasping force for three-finger tip-prehension grasps," ASME J. Mech., Trans., Automat. Des., vol. 110, pp. $405-413,1988$.

[16] J. C. Trinkle, "On the stability and instantaneous velocity of grasped frictionless objects," IEEE Trans. Robot. Automat., vol. 8, pp. 560-572, Oct. 1992.

[17] J. Kerr and B. Roth, "Analysis of multifingered hands," Int. J. Robot. Res., vol. 4, no. 4, pp. 3-17, 1986.

[18] Y.-C. Chen, I. D. Walker, and J. B. Cheatham, "Grasp synthesis for planar and solid objects," J. Robot. Syst., vol. 10, pp. 153-186, 1993.

[19] A. Bicchi, "On the closure properties of robotic grasping," Int. J. Robot. Res., vol. 14, no. 4, pp. 319-334, Aug. 1995.

[20] V. K. Varma and U. Tasch, "A new representation for a robot grasping quality measure," Robotica, vol. 13, pp. 287-295, 1995.

[21] Y. Nakamura, K. Nagai, and T. Yoshikawa, "Dynamics and stability in coordination of multiple robotic mechanisms," Int. J. Robot. Res., vol. 8, no. 2, pp. 44-59, 1989.

[22] J. Ponce, J. W. Burdick, and E. Rimon, "Computing the immobilizing three-finger grasps of planar objects," in Proc. 2nd Workshop on Comp. Kinematics, 1995.

[23] D. Prattichizzo, J. K. Salisbury, and A. Bicchi, "Contact and grasp robustness measures: Analysis and experiments," in Proc. 4th Int. Symp. Experimental Robotics, 1995, pp. 50-60. 
[24] M. R. Cutkosky and I. Kao, "Computing and controlling the compliance of a robotic hand," IEEE Trans. Robot. Automat., vol. 5, pp. 151-165, Apr. 1989.

[25] T. Patterson and H. Lipkin, "Structure of robot compliance," ASME J. Mech. Des., vol. 115, no. 3, pp. 576-580, 1993.

[26] Y.-C. Chou, V. Chandru, and M. M. Barash, "A mathematical approach to automatic configuration of machining fixtures: Analysis and synthesis," ASME J. Eng. Industry, vol. 111, pp. 299-306, 1989.

[27] S. P. Timoshenko and J. N. Goodier, Theory of Elasticity, 3rd ed. New York: McGraw-Hill, 1969.

[28] Q. Lin, "Mechanics and planning of workpiece fixturing and robotic grasping," Ph.D. dissertation, Dept. Mech. Eng., California Inst. Technol., Pasadena, May 1998.

[29] H. Hertz, "On the contact of rigid elastic solids and on hardness" (in English), in Misc. Papers by H. Hertz. London, U.K.: Macmillan, 1896.

[30] Y. E. Nesterov and A. S. Nemirovsky, Interior Point Polynomial Methods in Convex Programming: Theory and Applications. New York: Springer-Verlag, 1992.

[31] A. F. Van-Der-Stappen, C. Wentink, and M. H. Overmars, "Computing form-closure configurations," in Proc. Int. Conf. Robotics and Automation, 1999, pp. 1837-1842.

[32] E. Rimon and J. Burdick, "New bounds on the number of frictionless fingers required to immobilize planar objects," J. Robot. Syst., vol. 12, no. 6, pp. 433-451, 1995.

[33] Q. Lin, J. W. Burdick, and E. Rimon, "Minimum-deflection grasps and fixtures," in Proc. Int. Conf. Robotics and Automation, Leuven, Belgium, May 1998, pp. 3322-3328.

[34] C. A. Floudas and V. Visweswaran, "Quadratic optimization," in Handbook of Global Optimization, R. Horst and P. M. Pardalos, Eds. Norwell, MA: Kluwer, 1995.

[35] S. Vavasis, Nonlinear Optimization: Complexity Issues. Oxford, U.K.: Oxford Univ. Press, 1991.
Qiao Lin received the Ph.D. degree in mechanical engineering from the California Institute of Technology (Caltech), Pasadena, in 1998. His dissertation research involved automated grasp and fixture planning, with an emphasis on kinematics and mechanics of compliant grasps and fixtures.

He conducted postdoctoral research on design and fabrication of microelectromechanical systems (MEMS) at the Caltech Micromachining Laboratory from September 1998 to August 2000. He has been an Assistant Professor in the Department of Mechanical Engineering, Carnegie Mellon University, Pittsburgh, PA, since September 2000.

Joel W. Burdick received the B.S.M.E. degree from Duke University, Durham, NC, in 1981, and the Ph.D. degree in mechanical engineering from Stanford University, Stanford, CA, in 1988 .

He has been with the Department of Mechanical Engineering, California Institute of Technology, Pasadena, since 1988, where he is currently an Associate Professor. His research interests include robotic locomotion, grasping applications of robotics, and applied nonlinear control.

Dr. Burdick received the National Science Foundation Young Investigator Award, the Office of Naval Research Young Investigator Award, and the Feynman-Hughes Fellowship.

Elon Rimon, photograph and biography not available at the time of publication. 